\title{
Perspectives for future light source lattices incorporating yet uncommon magnets
}

\author{
S. C. Leemann* \\ MAX-lab, Lund University, S-22363 Lund, Sweden
}

A. Streun

SLS, Paul Scherrer Institute, CH-5232 Villigen, Switzerland

(Received 30 June 2010; published 3 March 2011)

\begin{abstract}
Although octupoles, decapoles, and longitudinal gradient bending magnets (LGB) have been studied for many years, they are not usually included in light source lattices. They can, however, be beneficial in order to realize ultralow emittance and attain sufficient dynamic aperture. We present methods for achieving ultralow emittance and discuss optimization of the nonlinear dynamics with multipoles. We demonstrate how control of amplitude-dependent tune shift makes octupoles a powerful tool for dynamic aperture optimization. Control of higher-order chromaticity by octupoles and decapoles is straightforward; however, since this turns out to be not quite as efficient in high-brightness lattices with low arc dispersion, we apply it to a conventional lattice to demonstrate the potential. This paper also illustrates how high-field LGBs can be used to build a compact, bright hard x-ray source. Finally, we demonstrate in detail the application of octupoles as integral components of the MAX IV $3 \mathrm{GeV}$ storage ring lattice.
\end{abstract}

DOI: 10.1103/PhysRevSTAB.14.030701

\section{INTRODUCTION}

Despite the rise of free-electron lasers, storage ringbased light sources remain the workhorses for research with synchrotron radiation. A new generation of rings (PETRA III [1], NSLS-II [2], MAX IV [3]) is characterized by a natural emittance $\leq 1 \mathrm{~nm}$ rad and significant impact of insertion devices on the radiation equilibrium. Lattices for an "ultimate light source" with emittance in the pm rad range providing fully diffraction-limited photon beams are being studied (see, for example, [4-8]).

In this paper we investigate concepts for ultralowemittance lattices and discuss the use of magnets which, although known and used in some high-energy physics lattices, are not yet common in light sources, namely, dipoles with longitudinal gradients, octupoles, and decapoles. The next section gives an overview of lattice design efforts to reach minimum emittance. The following section then introduces higher-order magnets used to optimize nonlinear beam dynamics in ultralow-emittance lattices. Finally, three lattices will be presented as case studies to demonstrate the benefits of using such magnets.

\section{LOW-EMITTANCE LATTICES}

The natural horizontal equilibrium emittance of a flat storage ring lattice [9] in practical units is

\footnotetext{
*simon.leemann@maxlab.lu.se

Published by American Physical Society under the terms of the Creative Commons Attribution 3.0 License. Further distribution of this work must maintain attribution to the author(s) and the published article's title, journal citation, and DOI.
}

PACS numbers: 29.20.db, 29.27.Bd, 29.27.Fh, 41.60.Ap

$$
\epsilon_{x o}[\mathrm{nmrad}]=1470(E[\mathrm{GeV}])^{2} \frac{I_{5}}{J_{x} I_{2}} \text { with } J_{x}=1-\frac{I_{4}}{I_{2}}
$$

and the radiation integrals

$$
\begin{gathered}
I_{2}=\oint h^{2} d s \quad I_{4}=\oint \eta\left(h^{3}+2 h b_{2}\right) d s \\
I_{5}=\oint h^{3} \mathcal{H} d s
\end{gathered}
$$

with $h=1 / \rho$ the orbit curvature, $b_{2}[>0]$ the (horizontally) focusing gradient, and $\mathcal{H}$ the dispersion's emittance,

$$
\mathcal{H}=\gamma_{x} \eta^{2}+2 \alpha_{x} \eta \eta^{\prime}+\beta_{x} \eta^{\prime 2}
$$

and $\alpha, \beta, \gamma$ the Twiss parameters and $\eta, \eta^{\prime}$ the dispersion and its slope.

\section{A. Theoretical minimum emittance}

Two cases for calculation of the theoretical minimum emittance (TME) of a bending magnet of homogenous curvature $h$ are usually considered (cf. Fig. 1): the center bending magnet $(\mathrm{CBM})$ located at the center of a periodic cell as used inside multibend achromats (MBA), and the end bending magnet $(\mathrm{EBM})$ where the dispersion is zero on one end as used in double bend achromats (DBA) or at the ends of an MBA facing the adjacent, dispersion-free straights [10]. Assuming an isomagnetic lattice, i.e., all bending magnets are of same type, with constant curvature $h$ and a bending angle $\Phi=h L$, the TME is obtained by solving integral $I_{5}$ over $\mathcal{H}$ from Eqs. (2) and (3) and minimizing the result with respect to the values of $\alpha_{x}$, $\beta_{x}, \eta$, and $\eta^{\prime}$ at some point [11-15]—cf. Appendix A for details. For small bending angles $\left(\Phi<20^{\circ}\right)$ the emittance can be written as 


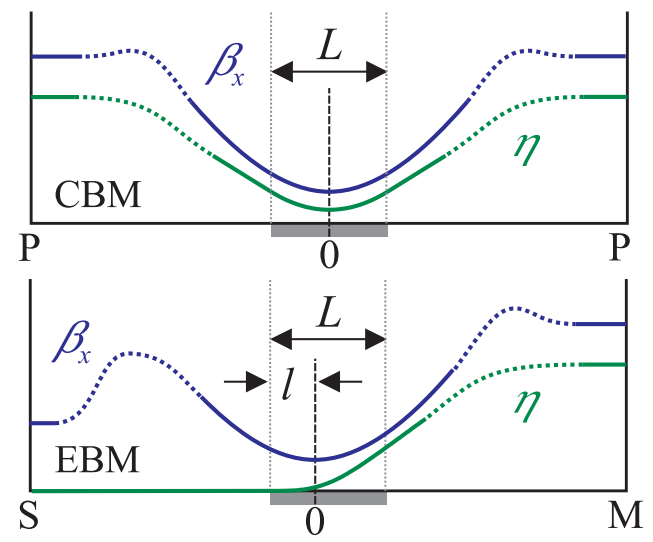

FIG. 1. Center bend and end bend cells.

$$
\epsilon_{x o}[\mathrm{pm} \mathrm{rad}]=\frac{7.8}{J_{x}}(E[\mathrm{GeV}])^{2}\left(\Phi\left[^{\circ}\right]\right)^{3} \frac{F}{12 \sqrt{15}},
$$

where the factor $F$ depends on the shape of the horizontal beta function and dispersion inside the bend. In the CBM the beam has a focus $\left(\alpha_{x o}=\eta_{o}^{\prime}=0\right)$ at the magnet center and the values of the beta function and dispersion at that focus have to be adjusted. For the EBM dispersion is zero at the entrance edge and thus fixed, then the distance $l$ of the focus (where $\alpha_{x}=0$ ) from the entrance edge and the beta function at that focus have to be adjusted. This results in the following matching conditions and TME values:

CBM: $\beta_{x o}^{\min }=\frac{1}{2 \sqrt{15}} L \quad \eta_{o}^{\min }=\frac{h L^{2}}{24} F^{\min }=1$

EBM: $\beta_{x o}^{\min }=\sqrt{\frac{3}{320}} L \quad l^{\min }=\frac{3}{8} L \quad F^{\min }=3$.

For deviations from the ideal matching conditions, expressed by parameters

$$
b=\frac{\beta_{x o}}{\beta_{x o}^{\min }} \quad \text { and } \begin{array}{cc}
d=\eta_{o} / \eta_{o}^{\min } & (\mathrm{CBM}) \\
\lambda=l / L & (\mathrm{EBM})
\end{array}
$$

ellipse equations are obtained [16] (cf. Appendix A) and shown in Fig. 2:

$$
\text { CBM: } \frac{5}{4}(d-1)^{2}+(b-F)^{2}=F^{2}-1
$$

EBM: $15(8 \lambda-3)^{2}+(3 b-F)^{2}=F^{2}-9$.

The cell phase advance $\mu$ can be calculated solely from the constraints that the slopes of dispersion and beta function have to disappear at the end points of the periodic cell "P" $(\mathrm{CBM})$ or at the point of mirror symmetry "M" (EBM) (cf. Fig. 1). In the EBM case, however, the optics from the straight center " $S$ " to the bending magnet is not determined, therefore we consider only the phase advance
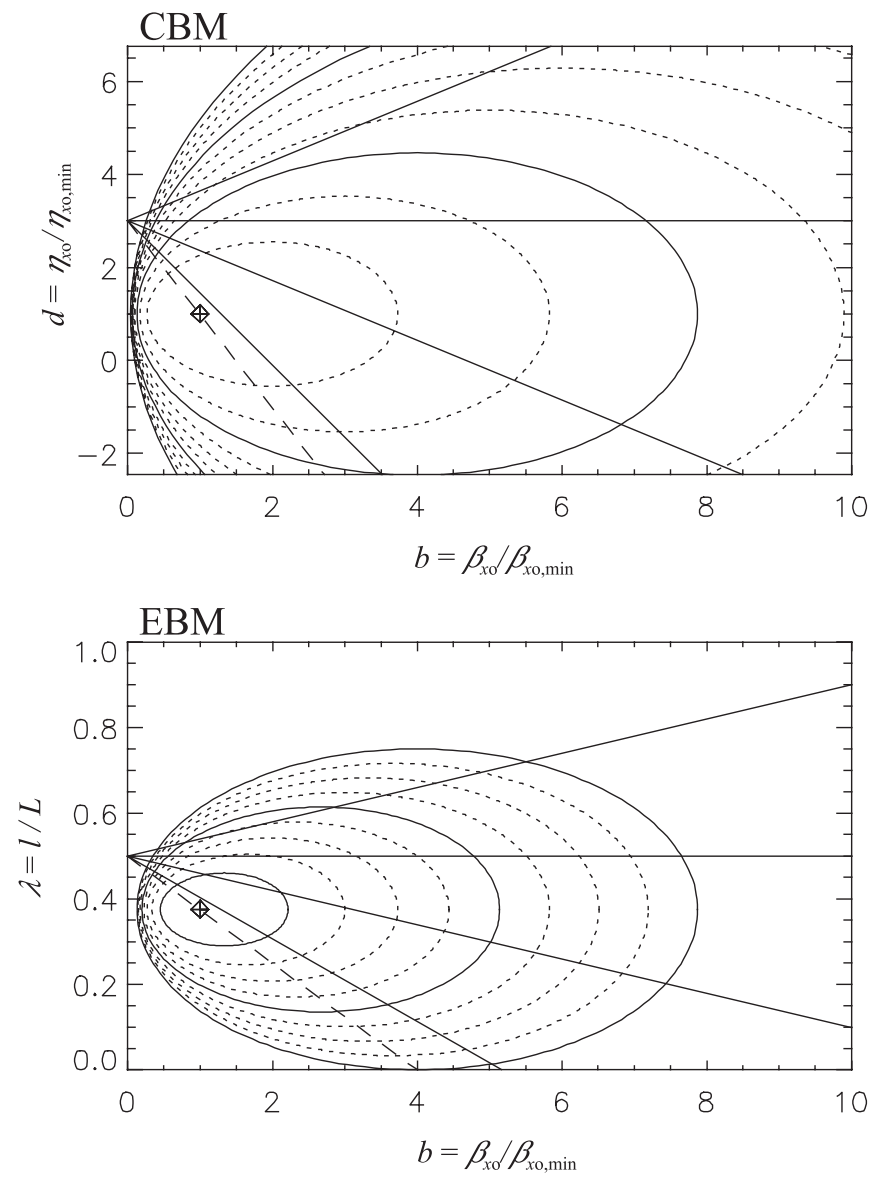

FIG. 2. Emittance of center (top) and end (bottom) bending magnets: Diamond symbols mark the TME conditions for $F^{\mathrm{min}}=1(\mathrm{CBM})$ and $F^{\mathrm{min}}=3(\mathrm{EBM})$. The elliptic isoemittance contours correspond to integer values up to $F=12$, where $F=$ 4, 8, 12 are solid, others dotted. The solid lines from upper to lower correspond to cell phase advances of $(3,4,5,6) \times 45^{\circ}$, the dashed line to $284.5^{\circ}$ as required in both cases to fulfill the TME condition.

between point " 0 " and its mirror image, whereas it is for the whole cell in the CBM case:

$$
\begin{aligned}
& \text { CBM: } \tan \frac{\mu}{2}=\frac{6}{\sqrt{15}} \frac{b}{(d-3)} \\
& \text { EBM: } \tan \frac{\mu}{2}=\frac{3}{\sqrt{15}} \frac{b}{(8 \lambda-4)} .
\end{aligned}
$$

It is interesting to note that fulfilling the TME conditions in both cases results in a phase advance of $284.5^{\circ}$, and thus an additional focus at point "P" or " $\mathrm{M}$ ". The DBA of the ELETTRA storage ring shown in Fig. 3 is nearest to the TME condition with $F=4.1$ and exhibits the additional focus to realize a phase advance of $\mu=216^{\circ}$ which requires additional drift spaces [17]. Because of limited circumference most light source lattices therefore use more relaxed optics with $\mu<180^{\circ}$ and correspondingly larger emittances. 


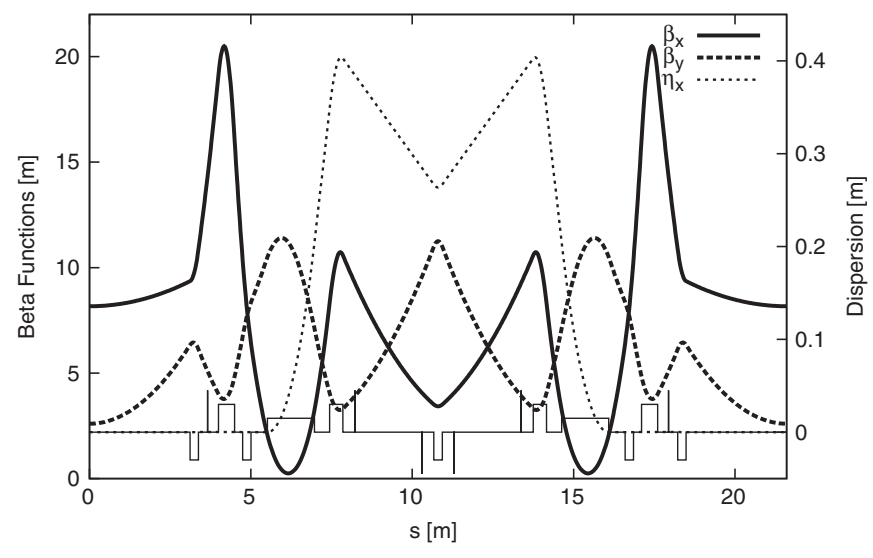

FIG. 3. The double bend achromat of the ELETTRA storage ring.

\section{B. Gradient bends}

Increasing the horizontal damping partition number $J_{x}$ has been done in many places (e.g. ALS [18], ELETTRA [17], ALBA [19]) to reduce the emittance at the expense of increased energy spread. This is achieved by introducing a vertically focusing gradient in the dipole magnets [cf. Eqs. (1) and (2)].

The reduction of the field at the orbit leads to longer bending magnets, but since the gradient also allows one to save vertically focusing quadrupoles, the net result is a reduction of machine circumference.

Beyond that, the lower field and correspondingly lower radiated power leads to a relative increase of the insertion devices' contribution to the radiation equilibrium and thus supports further reduction of emittance by dedicated damping wigglers and to some extent also by the users' insertion devices.

\section{Nondispersive straights}

Historically, dedicated light sources were planned with nondispersive straights in order to avoid synchrobetatron oscillations from cavities which were also located in straights and to minimize the impact of any longitudinal noise, i.e., rf jitter on the horizontal beam profile.

After both problems could be controlled by other means, mainly development of higher order mode (HOM) free or HOM damped cavities, and improvements of rf amplifiers, DBA lattices were tuned to allow dispersion in the straights in order to reduce the emittance, i.e., moving from the EBM towards a CBM optics (e.g. ESRF [20]) or were already designed with dispersive straights (e.g. SOLEIL [21]). However, since the dispersion contributes to beam width and divergence, the increased effective emittance as given by Eq. (B3) in Appendix B ultimately determines the brightness.

In very low $(<1 \mathrm{~nm} \mathrm{rad})$ emittance lattices (e.g. NSLS-II, MAX IV) insertion devices contribute significantly to the equilibrium emittance, leading to a further reduction only if the straight section dispersion is zero or very small, otherwise the emittance may easily increase; furthermore, the dispersion contribution to the effective emittance could become dominant. Therefore, ring-based light sources of the latest generation always have nondispersive straights.

\section{Longitudinal gradient bends}

In a CBM (cf. Fig. 1) the beta function and dispersion as well as $\mathcal{H}$ [cf. Eq. (3)] grow from the magnet center towards the edges. Thus, a homogenous field and curvature $h=B /(B \rho)$ is obviously not the optimum solution in order to minimize the radiation integral $I_{5}$ and the emittance [cf. Eqs. (1) and (2)]. Instead, introducing a longitudinal field gradient for highest curvature at the bend center and lowest at the edges while keeping the bending angle

$$
\Phi=\int_{L} h(s) d s
$$

constant, may compensate for the variation of $\mathcal{H}$ and provide an emittance lower than the TME. Finding a function $h(s)$ to minimize Eq. (1) under the constraint of Eq. (9) represents an isoperimetric variational problem, which however cannot be solved analytically in general. Instead, numerical minimization [22] or semianalytical minimization assuming special functions for $h(s)$ [23,24] have been carried out. Here, we want to briefly investigate two methods to build a lattice cell based on an LGB.

(i) Starting at the center of a CBM, we assume a maximum field value and set values for $\beta_{x}$ and $\eta$, which will give the initial value $\mathcal{H}_{o}$. Propagating the parameters through a slice of constant field and length $\Delta s$ will give another, usually a larger value of $\mathcal{H}_{1}$ at the end of the slice. So, the field of the next slice is scaled down by

$$
B_{1}=B_{o} \sqrt[3]{\frac{\mathcal{H}_{o}}{\mathcal{H}_{1}}}
$$

and so on, in order to get the same contribution to $I_{5}$ from each slice. In this way the calculation continues until the desired angle of deflection of the LGB has been reached.

Since the field will become lower when moving from the center to the edge, there is room to introduce a gradient of positive or negative sign. Assuming a magnet pole half width $w$ with a maximum pole-tip field $B_{\max }$, the available gradient is thus limited by

$$
\left|B(s)^{\prime}\right| \leq \min \left\{\frac{B_{\max }-B(s)}{w} ; \frac{B(s)}{w}\right\} .
$$

The second limitation corresponds to a half-quadrupole of radius $w$ as a limiting case: for $B^{\prime}>B / w$ the bending magnet would become an off-centered quadrupole and require four poles.

An example is shown in Fig. 4: The field decay from center to edge compensates for the growth of $\mathcal{H}$. At 

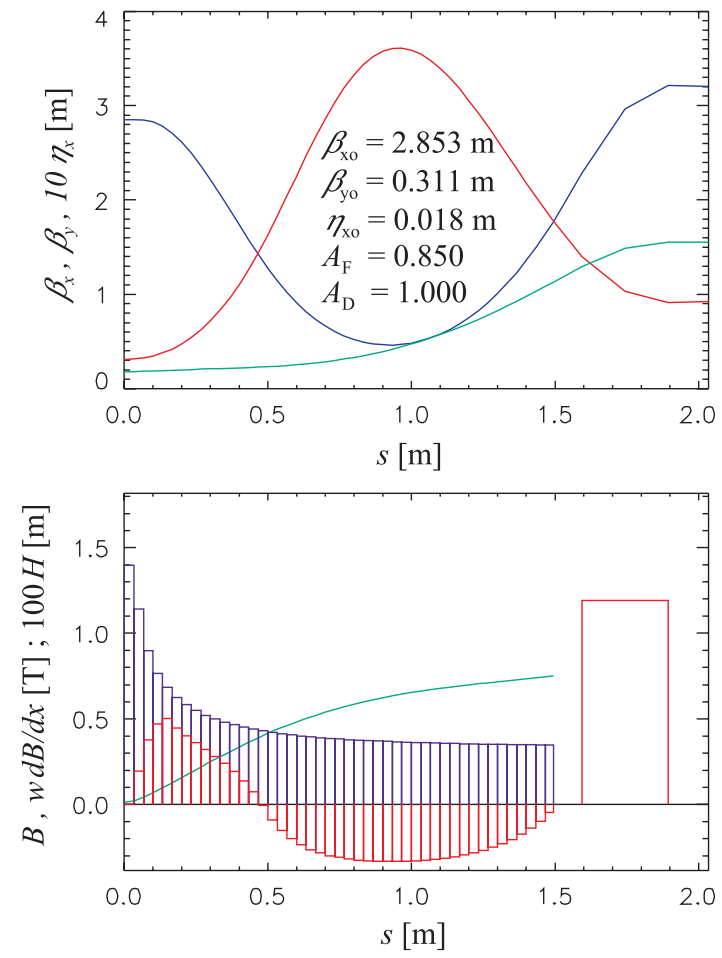

FIG. 4. Example for a longitudinal gradient bending magnet: The upper plot shows, from center to edge, the optical functions of the LGB, $\beta_{x}$ in blue, $\beta_{y}$ in red, $\eta$ in green. The lower plot shows the field $B$ (blue) and the additional field due to the gradient, $w B^{\prime}$ (red) for each slice of the magnet. The function $\mathcal{H}$ is shown in green. The rectangle at the right is a quadrupole to perform the periodic matching. Initial beam parameters are displayed in the upper plot. The attenuation factors $\left(A_{\mathrm{F}, \mathrm{D}}\right)$ indicate how much of the available gradient was used horizontally and vertically.

$2.4 \mathrm{GeV}$, a periodic cell incorporating this LGB, as shown in Fig. 5 (top), would provide $10^{\circ}$ of deflection at a length of $4.1 \mathrm{~m}$, and, using a damping partition of $J_{x}=1.9$, an emittance of $\epsilon_{x o}=0.7 \mathrm{~nm} \mathrm{rad}$, which is about $30 \%$ lower than the TME of a $10^{\circ} \mathrm{CBM}$. Chromaticity corrections with sextupole components incorporated in the LGB and the end quads results in large dynamic aperture (DA) of the ideal cell.

However, the disadvantage of this LGB is its length, the complex pole shape, and the limited tunability and flexibility. The same space could as well accommodate two cells using standard magnets as shown in Fig. 5 (bottom), which can easily be tuned to provide exactly the same or even better performance, because the factor 8 gained in emittance by just splitting the center bend into two bends provides leeway to relax and tune the optics. So, this LGB concept appears inferior to a multibend achromat (MBA) scheme.

(ii) A more promising method to exploit an LGB cell is based on matching optical functions to a superbend, which has a longitudinal gradient by nature: a superbend has a
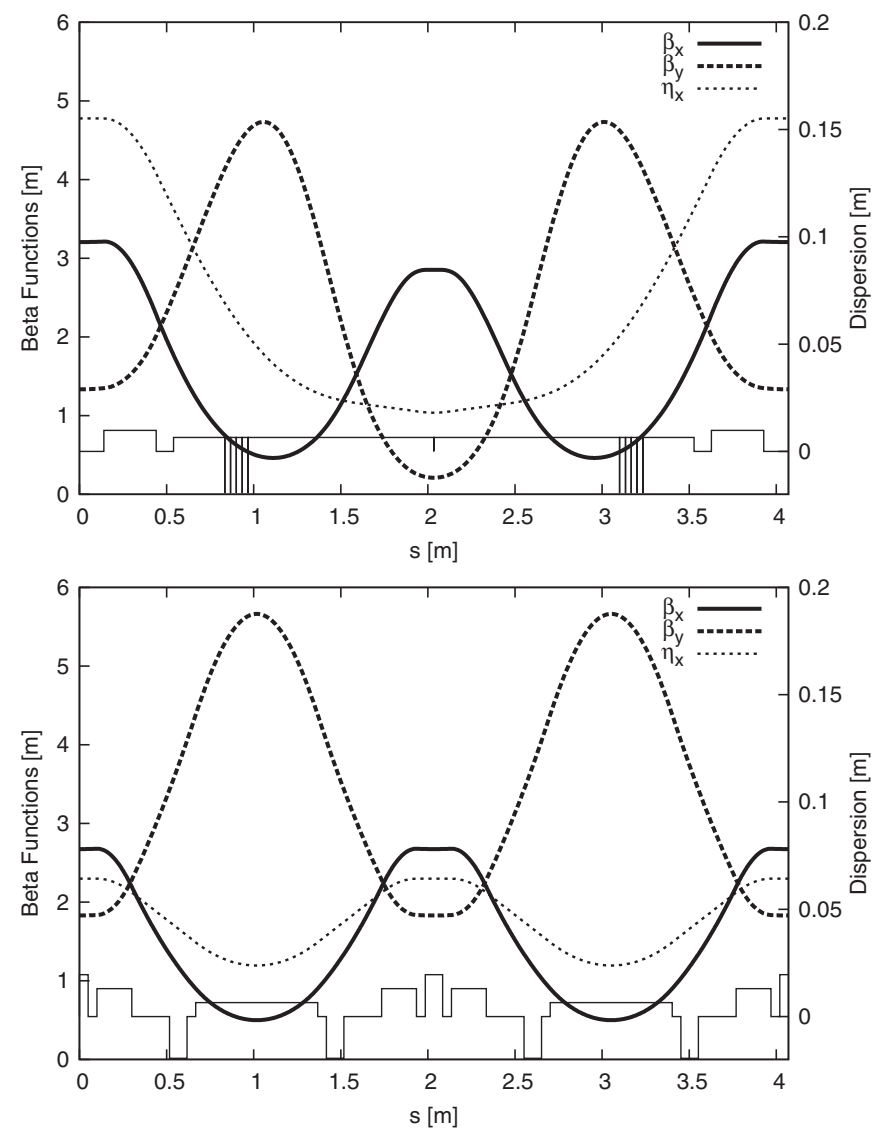

FIG. 5. Periodic cell incorporating a longitudinal gradient bend (top) and a double cell with homogenous bends for comparison (bottom). Both cells have identical length, angle, $J_{x}$, and emittance.

central high-field region for production of hard x rays, and lower field elsewhere to keep the radiation losses low. For example, the superbends of the ALS have a longitudinal field profile which can be roughly approximated by a Gaussian of about 5.7 T peak and $0.18 \mathrm{~m}$ FWHM [25] (cf. Fig. 15 in Sec. IV B). Using a superbend as a CBM, the center beta function and dispersion for lowest emittance can be found numerically or even analytically for further simplified cases, and emittance ellipses analogous to the ones shown in Fig. 2 and cell phase advances can be calculated [24]. The resulting values of beta function and dispersion, however, scale inversely with the central field strength, i.e., they become very small. The ALS superbend would provide an emittance as low as $40 \%$ of the TME of a homogenous CBM of same deflection angle for $\beta_{x o} \approx 2.5 \mathrm{~cm}, \eta_{o} \approx 0.8 \mathrm{~mm}$ at its center. This sharp focus leads to a large beta function at the adjacent quadrupoles and thus to large chromaticities and subsequent DA problems.

Therefore, the LGB cell would not be tuned for ultimate low emittance, but to an emittance which is comparable to the TME of a homogenous CBM. In case of the ALS 
superbend, this would be the case for a 5 times larger value of $\beta_{x o}$ along with substantially relaxed optics as well as reduced cell phase advance [also cf. Fig. 2 (top)].

A more detailed elaboration of this concept is given in Sec. IV B presenting a compact brilliant hard x-ray source based on super-LGB cells.

\section{E. Multibend achromats}

Low-emittance lattices using either LGBs or homogenous bending magnets in TME mode will require much space: the TME optics requires "empty" cells alternating with the bending magnets to accommodate the additional focus required for matching, and since these empty cells have high dispersion, they cannot be used for undulators. A normal-conducting LGB will be very long to match the decreasing field strength to the increasing $\mathcal{H}$ function (superconducting LGB lattices however could be rather compact).

For a given circumference of the lattice, the multibend achromat (MBA) lattice is obtained as a third solution by virtually dividing each bending magnet in two, using the space which was used for empty cells in the TME case, and decreasing field in the LGB case for another cell, thus exploiting the cubic dependence of emittance on bending angle per magnet. The factor 8 in emittance thus gained may be invested to allow for a very moderate phase advance per cell $\left(\approx 100^{\circ}\right.$, cf. Fig. 2 top $)$ and realize a rather relaxed low-emittance lattice with moderate chromaticities and hence alleviated DA optimization [26-30].

A small bending angle per magnet and short cells result in small peak values of dispersion and horizontal beta function and thus in small horizontal apertures, given by

$$
a_{x} \geq \max \left\{2 \eta(s) \delta_{\mathrm{acc}} ; \sqrt{A_{x} \beta(s)}\right\}
$$

with $A_{x}$ the horizontal acceptance, i.e., the betatron amplitude required to capture the injected beam, and $\delta_{\text {acc }}$ the momentum acceptance (MA) required to provide sufficient Touschek lifetime. The vertical aperture is small anyway due to the presence of undulators with very narrow gaps. Small magnet apertures, however, allow high multipole gradients and hence compact magnets which further supports a compact arc $[29,30]$.

A similar concept had been developed earlier guided by the concept to distribute the bending around the machine and combining dipolar, quadrupolar, and sextupolar fields in the same magnets [31]. The idea was to compensate the chromaticity where it is created in order to minimize the chromatic beta beat. These lattices were found to provide large DA and very large MA. Application to booster synchrotrons [32] resulted in economic machines providing low emittance and low power consumption due to the low stored field energy of the small magnets. Booster synchrotrons of this type are perfectly suited to top-up operation and operate successfully in several places [33-35].

So, following the MBA approach, slim and rather inexpensive lattices can be built which either become very compact or allow very small emittance. However, two problems have to be solved: (i) Small apertures require a small emittance of the injected beam which requires injection from either a linear accelerator (MAX IV) or from a low-emittance synchrotron based on the same MBA concept [36]. (ii) The small peak dispersion results in high gradients of the sextupoles used for chromaticity compensation. This is not much of a complication for the magnet design, since the small aperture allows high gradients, but it leads to challenges of nonlinear dynamics optimization even for a rather relaxed lattice, which will be discussed in the next section.

Nevertheless, we believe the MBA approach as applied to the design of MAX IV [3] to be superior to TME and LGB for building an ultralow-emittance lattice within a limited circumference.

\section{OPTIMIZING THE NONLINEAR DYNAMICS}

Usually ultralow-emittance lattices have many strong quadrupole magnets in order to provide a horizontal focus in each dipole. A characteristic of these strong focusing lattices is the large negative natural chromaticity which needs to be corrected with strong sextupoles. Beside the chromatic sextupoles used to tune the two first-order chromatic terms $\left(h_{11001}\right.$ driving $\xi_{x}, h_{00111}$ driving $\left.\xi_{y}\right)$, harmonic sextupoles are introduced to minimize the remaining firstorder sextupole driving terms: three chromatic terms (second-order dispersion $\partial \eta_{x} / \partial \delta$ and momentumdependent beta beat $\partial \beta_{x, y} / \partial \delta$ ) and five geometric terms (driving the resonances $Q_{x}, 3 Q_{x}$, and $Q_{x} \pm 2 Q_{y}$ ). Properly tuning these terms alone will however not ensure large DA and MA: second-order effects including amplitudedependent tune shifts $\left(\partial \nu_{x, y} / \partial J_{x, y}\right)$ and second-order chromaticity $\left(\xi_{x, y}^{(2)}=\partial^{2} \nu_{x, y} / 2 \partial \delta^{2}\right)$, along with even higherorder effects, shape the tune footprint and thus determine the dynamic performance of the lattice.

The systematic nonlinear dynamics approach is to install sextupoles at locations separated by suitable betatron phases and adjust the strengths of the different sextupole families in such a way as to correct chromatic behavior while minimizing resonance driving terms $[37,38]$. Ideal placement is however often not possible leading to increased required sextupole strength and degeneration of driving terms.

In addition, the weak dispersion of low-emittance lattices requires very strong sextupole magnets. Adjusting amplitude-dependent tune shifts (ADTS) and/or secondorder chromaticities requires even more sextupole strength because these corrections are only applied by the sextupoles in second order. This results in a situation where 
many very strong sextupoles have to be installed to keep linear chromaticity and first-order resonance driving terms in check. Even if the applied sextupole strengths are adjusted in such a way that resonance driving terms are well compensated in the ideal bare lattice, this is a complicated and very delicate balance that is easily disturbed by insertion devices, alignment errors, and higher-order multipoles that all exist in real light source storage rings. An example for such a situation will be given in Sec. IVA where a modern ultralow-emittance storage ring design reached a dynamic performance limit due to the unavoidable trade-off between strength/number of installed sextupoles and desired DA/MA.

One possible remedy for this problem is-although not yet common in light sources-the use of higher-order magnets such as octupoles and decapoles where ADTS and higher-order chromatic behavior are manipulated to first order [39]. If installed at the proper locations (cf. below) octupole and decapole magnets can be operated at comparably low strength yet still allow sufficient compensation of sextupole effects. In addition, the freedom to choose magnitude and direction of tune shifts in phase space opens up the interesting possibility of tailoring dynamic behavior of a lattice $[40,41]$ to best suit its resonance environment in phase space: ADTS can be strongly suppressed so the tune remains close to the working point even for large excursions from the design orbit (for example, after injection); quadratic and cubic chromaticities can be shaped in such a way that chromatic tune shifts are "wrapped up" around the design working point thus avoiding resonance crossing even for severe off-momentum particles (for example, in extreme Touschek scattering events).

This is an intriguing approach because it relieves lattice designers of the constraint to keep sextupoles weak in order not to generate large higher-order tune shifts. If octupoles and decapoles are available, the lattice designer can adjust sextupoles mainly for first-order terms while the weak octupoles and decapoles are sufficient to compensate the higher-order sextupole terms. As long as the octupoles and decapoles remain comparably weak, they do not drive strong higher-order terms which would force lattice designers into a run-away cycle of even higher-order compensation.

\section{A. Octupoles}

The effect of inserting an octupole in a bare sextupole lattice can be expressed by evaluating the octupole Hamiltonian and including off-momentum particles as a perturbation. This derivation is shown in Appendix C. The resulting Hamiltonian can be expressed in the resonance basis [cf. Eq. (C10)] which allows immediate identification of the octupolar effects.

Because the five chromatic and amplitude-dependent tune shifts in the first-order octupole Hamiltonian [cf. Eqs. (C18)-(C21)] are determined solely by the octupole strength and the optical functions at the location of the octupole, it is straightforward to use an octupole to compensate the tune shifts resulting as second-order effects of a bare sextupole lattice. Define a vector containing amplitude-dependent and secondorder chromatic tune shifts:

$$
\overrightarrow{\delta \nu}=\left(\begin{array}{c}
\partial \Delta \nu_{x} / \partial J_{x} \\
\partial \Delta \nu_{y} / \partial J_{y} \\
\partial \Delta \nu_{x} / \partial J_{y} \\
\partial^{2} \Delta \nu_{x} / \partial \delta^{2} \\
\partial^{2} \Delta \nu_{y} / \partial \delta^{2}
\end{array}\right)
$$

Note that this vector is nonzero even for a bare sextupole lattice without any octupoles as a result of higher-order effects of the sextupoles. In fact, the chromatic terms are already nonzero for a bare quadrupole lattice [37]. Tune shifts resulting from octupoles in the lattice can then be expressed with the simple vector equation,

$$
\delta \vec{\nu}_{\text {oct }}=\mathbf{B}_{\text {oct }} \vec{b}_{4},
$$

where $\vec{b}_{4}$ is a vector with the total octupole strengths for each octupole family $j \in 1, \ldots, N_{\text {oct }}$ and $\mathbf{B}_{\text {oct }}$ is $5 \times N_{\text {oct }}$ matrix that contains the optical functions evaluated at the locations of the octupoles (as derived in Appendix C):

$$
\mathbf{B}_{\text {oct }}=\frac{3}{8 \pi}\left(\begin{array}{ccc}
\left(\beta_{x}\right)_{1}^{2} & \cdots & \left(\beta_{x}\right)_{N_{\text {oct }}}^{2} \\
-2\left(\beta_{x} \beta_{y}\right)_{1} & \cdots & -2\left(\beta_{x} \beta_{y}\right)_{N_{\mathrm{oct}}} \\
\left(\beta_{y}\right)_{1}^{2} & \cdots & \left(\beta_{y}\right)_{N_{\mathrm{oct}}} \\
4\left(\eta^{2} \beta_{x}\right)_{1} & \cdots & 4\left(\eta^{2} \beta_{x}\right)_{N_{\mathrm{oct}}} \\
-4\left(\eta^{2} \beta_{y}\right)_{1} & \cdots & -4\left(\eta^{2} \beta_{y}\right)_{N_{\mathrm{oct}}}
\end{array}\right) .
$$

Equation (12) is a linear system which can be inverted so an octupole setting $\vec{b}_{4}$ is found that cancels the tune shifts of the bare sextupole lattice $\delta \vec{\nu}$. Unless five octupole families are used, $\mathbf{B}_{\text {oct }}$ is not a square matrix and the linear system has to be solved using pseudoinversion (e.g. singular value decomposition). If fewer than five families are available, the resulting tune shift $\delta \vec{\nu}$ will be minimized; if more than five families are used the tune shifts can be canceled exactly, however, using the minimum total octupole strength $\left\|\vec{b}_{4}\right\|$. Because of higher-order effects over which one lacks direct control, it is often not desirable to suppress first-order ADTS entirely. Instead, nonzero values are chosen in order to minimize the resulting overall ADTS including higher orders over a certain range of interest (for example, within the physical aperture). Examples for such an optimization will be presented in Secs. IVA and IV B.

In order to use octupoles efficiently, they have to be placed properly with respect to the optical functions $\beta_{x, y}$ and $\eta$. In mathematical terms the inversion of Eq. (12) requires the matrix $\mathbf{B}_{\text {oct }}$ to have a rank of 5. In practical 
terms it can be understood that a chromatic octupole family $j$ needs nonzero dispersion $\eta_{j} \neq 0$ in order to counteract the second-order chromaticity; in fact, ensuring these octupoles are installed at locations where $\eta_{j}$ is very large results in lower required octupole strength. Likewise an octupole family intended to correct horizontal (or vertical) tune shifts needs to be installed at a location where $\beta_{x} \gg \beta_{y}\left(\right.$ or $\beta_{y} \gg \beta_{x}$ ). For the case of the coupling term $\partial \Delta \nu_{x} / \partial J_{y}=\partial \Delta \nu_{y} / \partial J_{x}$ an ideal location of the family would be where $\beta_{x} \approx \beta_{y}$. Making sure placement of the octupoles is chosen properly increases the leverage of each octupole family to fight a specific driving term. An interesting case to consider is when octupoles used to fight ADTS see large dispersion. This can create large chromatic tune shifts which then have to be counteracted again by the chromatic octupoles. Ideally, one would therefore choose dispersion-free locations for the placement of the octupole families used to counteract ADTS. This separation of function among different octupole families helps to ensure the resulting octupole corrections strengths remain low.

\section{B. Decapoles}

Decapoles can be treated analogously to octupoles. The decapole Hamiltonian has been expanded in Appendix D. It contains five terms that do not carry phases and therefore drive tune shifts. These terms are listed in Eqs. (D4)-(D7). Of practical interest is the use of decapoles to adjust thirdorder chromaticity $\xi_{x, y}^{(3)}$ in order to increase MA. This is especially important if suppression of coupled bunch instabilities enforces large positive values of linear chromaticity. In this case, introducing negative third-order chromaticity can reduce the chromatic tune footprint in order to avoid resonance crossings and thus increase the lattice MA.

For this purpose an approach similar to Eq. (12) is taken: the linear system

$$
\vec{\xi}^{(3)}=\mathbf{B}_{\mathrm{dec}} \vec{b}_{5}
$$

where $\vec{b}_{5}$ is a vector with the total decapole strengths for each decapole family $j \in 1, \ldots, N_{\text {dec }}, \vec{\xi}^{(3)}$ is a vector containing the third-order chromaticities:

$$
\vec{\xi}^{(3)}=\left(\begin{array}{l}
\partial^{3} \Delta \nu_{x} / \partial \delta^{3} \\
\partial^{3} \Delta \nu_{y} / \partial \delta^{3}
\end{array}\right),
$$

and $\mathbf{B}_{\mathrm{dec}}$ is a $2 \times N_{\mathrm{dec}}$ matrix that contains the optical functions evaluated at the locations of the decapole (as derived in Appendix D)

$$
\mathbf{B}_{\mathrm{dec}}=\frac{6}{\pi}\left(\begin{array}{ccc}
\left(\eta^{3} \beta_{x}\right)_{1} & \cdots & \left(\eta^{3} \beta_{x}\right)_{N_{\mathrm{dec}}} \\
-\left(\eta^{3} \beta_{y}\right)_{1} & \cdots & -\left(\eta^{3} \beta_{y}\right)_{N_{\mathrm{dec}}}
\end{array}\right) .
$$

Two families of dispersive decapoles are sufficient to set the third-order chromaticities. Ideally, each family would be installed at a location maximizing $\eta^{3} \beta_{x}$ and $\eta^{3} \beta_{y}$, respectively.

\section{LATTICE STUDIES}

This section will detail three example cases for the use of LGBs and octupoles in storage ring-based synchrotron light sources. The first case is the actual MAX IV $3 \mathrm{GeV}$ storage ring lattice which contains octupoles as integral components of the lattice. The second case is a test lattice designed to demonstrate the capabilities of the LGB; it also includes octupoles. The third case uses an operating storage ring lattice to demonstrate second-order chromaticity correction with octupoles.

\section{A. The MAX IV $3 \mathrm{GeV}$ storage ring lattice}

The MAX IV $3 \mathrm{GeV}$ storage ring is an excellent example for the substantial performance increase that can be achieved by using octupoles in a light source lattice. The MAX IV $3 \mathrm{GeV}$ storage ring lattice design is detailed elsewhere [3] and only a very brief overview will be given here. The storage ring lattice was tailored to match the requirements of state-of-the-art x-ray insertion devices: a $528 \mathrm{~m}$ circumference multibend achromat lattice with 20 -fold periodicity that achieves an ultralow emittance of $0.33 \mathrm{~nm}$ rad for the bare lattice; a reduction by $50 \%$ can be achieved if all straights are occupied with insertion devices. This lattice supplies users with 19 nondispersive $5 \mathrm{~m}$ straight section for insertion devices with beam sizes $\sigma_{x}<$ $60 \mu \mathrm{m}$ and $\sigma_{y}<6 \mu \mathrm{m}$ (bare lattice) at the center of these straights.

The crucial aspect of the lattice is the multibend achromat design with the underlying sevenfold unit cell periodicity. The many weak gradient dipoles with interleaved focusing quadrupoles result in small dispersion and, hence, ultralow emittance. A schematic of one achromat is given in Fig. 6. Although the linear optics can be considered relaxed, the resulting emittance is so low that beam lines will be operating in a vertically diffraction-limited regime. Further advantages of the lattice are the wide tunability of the beta function in the long straights (making local matching and compensation of insertion devices a feasible option) and the short straight sections in the dispersionsuppressing matching cell which can house rf cavities

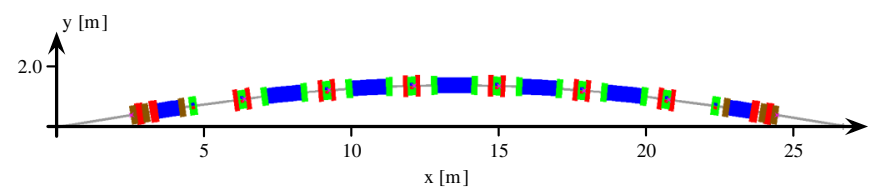

FIG. 6. Schematic of one of the 20 achromats of the MAX IV $3 \mathrm{GeV}$ storage ring. Magnets indicated are gradient dipoles (blue), focusing quadrupoles (red), sextupoles (green), and octupoles (brown). The basic structure of five unit cells flanked on either side by a matching cell can be recognized. 
therefore keeping all but one long straight (for injection) available for insertion devices.

\section{Description of the problem}

Originally, the limitation of this design in terms of nonlinear beam dynamics lay in the sextupole flexibility. Because of space constraints it was initially considered to integrate sextupoles into the gradient dipoles and the interleaved focusing quadrupoles. Besides resulting in a very compact and simple design, this approach has the advantage that it puts sextupoles at optimum location in terms of the beta functions thus reducing required sextupole strength. However, this approach also carries two distinct disadvantages: first, dispersion in the unit cell dipoles is low thus requiring stronger defocusing sextupole components in the gradient bends; second, integrating the sextupole component in gradient bends and quadrupoles technically limits the sextupole strength and removes tuning flexibility. In principle, back-leg windings can be used to adjust the sextupole component in the combinedfunction magnets, but this has the disadvantage that exciting these windings induces also a dipole field.

Instead, it was decided to install discrete sextupoles in the lattice. Defocusing sextupoles now flank the gradient dipoles and focusing sextupoles have been inserted at the location of the focusing quadrupoles [42] (cf. Fig. 6). This puts the focusing sextupoles at ideal locations in terms of dispersion and beta functions, but limits the number of focusing sextupole families to three. The defocusing sextupoles see less dispersion and a relatively low vertical beta function, but the large number of magnets mitigates the weaker leverage. However, due to the symmetry of the lattice only two to three defocusing families are feasible. Figure 7 shows the optical functions within the achromat and the positions of the magnets.

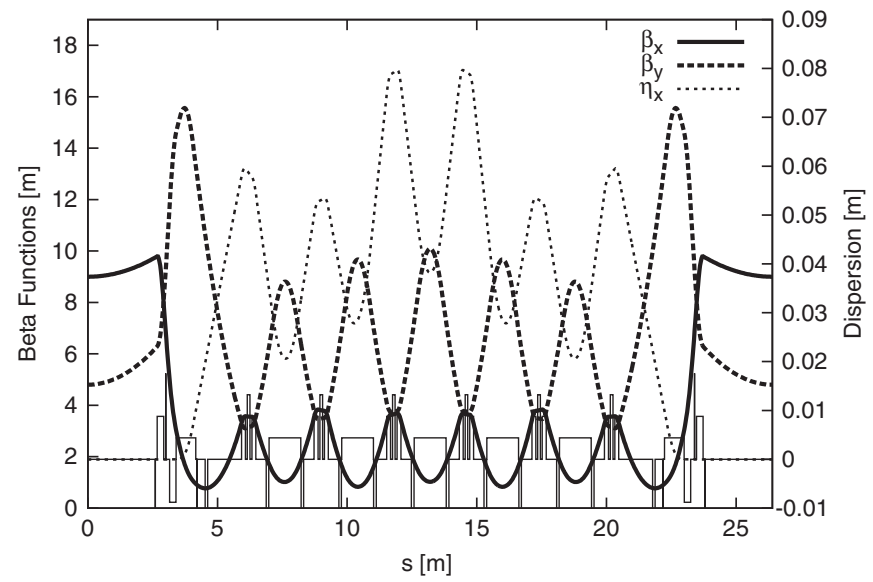

FIG. 7. Beta functions $\beta_{x}, \beta_{y}$ and dispersion $\eta_{x}$ for one achromat of the MAX IV $3 \mathrm{GeV}$ storage ring. The position of the dipoles, quadrupoles, and sextupoles are indicated at the bottom.

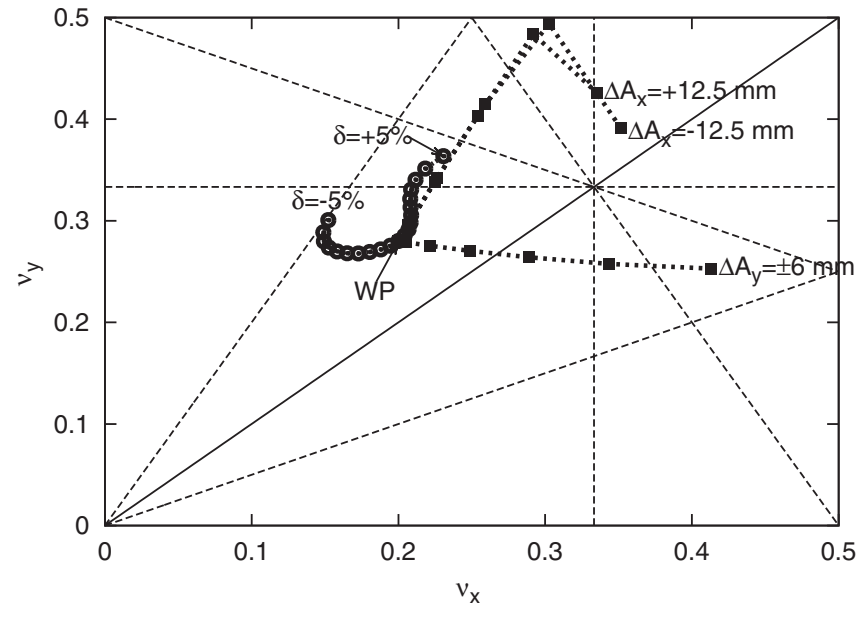

FIG. 8. Fractional tune space and tune shifts for the MAX IV $3 \mathrm{GeV}$ storage ring lattice with only five sextupole families. The working point at $42.20 / 14.28$ is indicated. For chromatic tune shifts $\delta$ the dots indicate $\pm 0.5 \%$ steps; for ADTS $\Delta A_{x}\left[\Delta A_{y}\right]$ black boxes indicate steps of $\pm 2.5 \mathrm{~mm}[ \pm 1.0 \mathrm{~mm}]$. Fractional tunes $\nu_{y}>0.5$ appear as mirror tunes $\left(1-\nu_{y}\right)$ in the diagram. Tracking was performed with OPA and results were verified with TRACY-3.

The sextupole settings for the MAX IV $3 \mathrm{GeV}$ storage ring were obtained by adjusting the linear chromaticity $\xi_{x, y}=+1.0$ and minimizing the first-order resonance driving terms. This original global minimization of driving terms resulted in the tune footprint displayed in Fig. 8. Unfortunately, the chromatic tune footprint was large and crossed the third-order resonance $3 \nu_{y}=43$ already for $\delta=+3.5 \%$ thus potentially limiting MA. In addition, the ADTS achieved in this manner were unacceptably high: large horizontal amplitudes (as expected during injection) would push the vertical tune to the half-integer resonance $2 \nu_{y}=29$; vertical amplitudes were limited at $<4 \mathrm{~mm}$ because of the third-integer resonance $3 \nu_{x}=127$.

The weights were subsequently changed in the Hamiltonian minimizer in order to suppress chromatic tune shifts. This did, however, not lead to the desired results. Instead the sextupole strengths were tweaked manually (away from the global minimum) to specifically tailor the quadratic chromaticity in such a way as to "wrapup" the chromatic tune shifts around the working point. The results are displayed in Fig. 9. The chromatic tune shifts for momentum deviations up to $\pm 5 \%$ have been compressed within an area of less than $0.07 \times 0.05$ in tune space keeping off-momentum particles clear of previously encountered low-order resonances.

However, ADTS remained too large resulting in the crossing of several potentially harmful resonances. Tracking with TRACY-3 [43] was used to verify OPA [44] results and to perform frequency map analysis [45]. A diffusion map generated by TRACY-3 [46] (displayed in Fig. 10) confirms these resonance crossings. Almost the entire area within the DA shows increased diffusion. The 


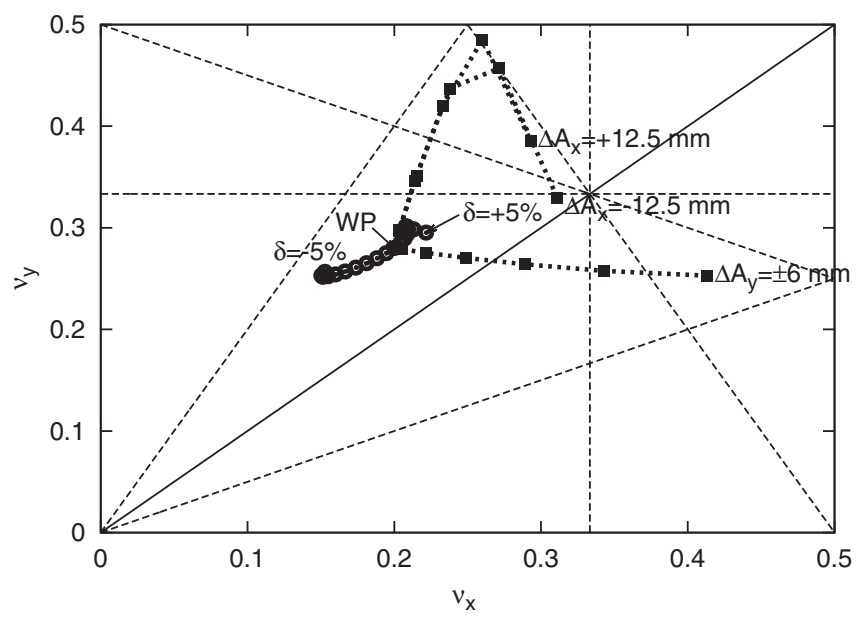

FIG. 9. Fractional tune space and tune shifts for the MAX IV $3 \mathrm{GeV}$ storage ring lattice with only five sextupole families. Fractional tunes $\nu_{y}>0.5$ appear as mirror tunes $\left(1-\nu_{y}\right)$ in the diagram. The sextupole settings have been adjusted to reduce chromatic tune shifts and, hence, increase lattice MA (cf. Fig. 8). The ADTS remain however too large. Tracking was performed with OPA and results were verified with TRACY-3.

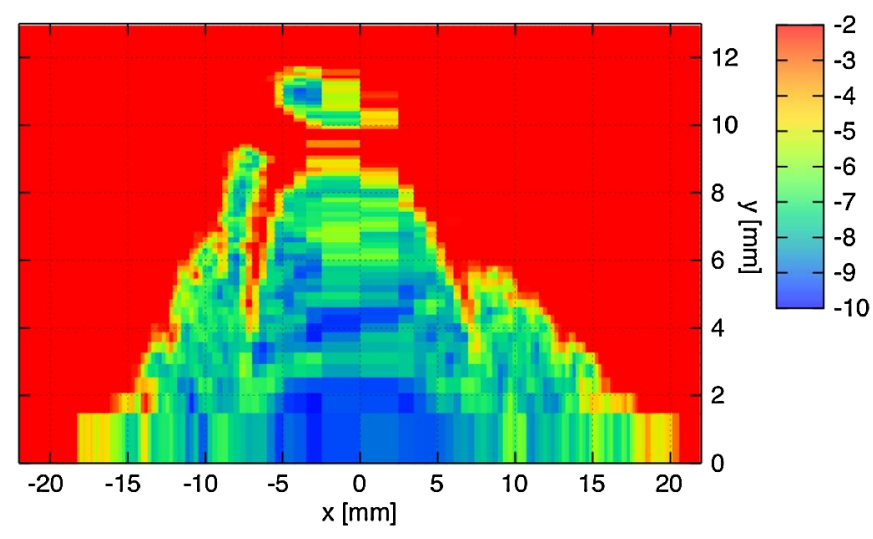

FIG. 10. Diffusion map (2000 turns) at the center of the $5 \mathrm{~m}$ long straight section for the MAX IV $3 \mathrm{GeV}$ storage ring lattice with only five sextupole families. The scale is logarithmic in tune shift from low (blue) to high (red).

two diffusion "protrusions" are caused by the half-integer resonances $2 \nu_{x}=85$ and $2 \nu_{y}=29$. In addition to insufficient DA and the increased diffusion within the DA (especially within the required stay-clear aperture of $[ \pm 8 \mathrm{~mm}] \times[ \pm 2.5 \mathrm{~mm}])$, the islandlike structure for large vertical excursions is unsatisfactory. In fact, most of the available vertical DA is wasted considering narrow-gap invacuum insertion devices will be installed. Instead, it would be desirable to trade off this excess vertical DA for additional horizontal DA.

\section{Octupole solution}

For the MAX IV $3 \mathrm{GeV}$ storage ring, it was therefore decided to reduce the ADTS in order to increase the DA and reduce sources of diffusion within. Because adding additional sextupole families was not feasible and reducing the ADTS with the existing sextupole families was only possible at the expense of increasing the chromatic tune shift, it was decided to introduce dedicated octupoles into the lattice [47].

Since the chromatic tune shift had been sufficiently tailored to the tune space environment of the working point, only harmonic octupoles were required [48]. A first family was installed in the long straight section immediately after the last focusing quadrupole. This is the only location in the achromat with the proper beta function ratio $\left(\beta_{x}>\beta_{y}\right)$ to counteract the $\partial \Delta \nu_{x} / \partial J_{x}$ tune shift. The tune shift $\partial \Delta \nu_{y} / \partial J_{y}$ requires a large $\beta_{y} / \beta_{x}$ ratio. The location in the achromat that maximizes this ratio is found between the last defocusing sextupole and the matching dipole. Because this dipole opens up the dispersion, it was decided to move the octupole right next to the dipole. Finally, a location with roughly equal beta function values was needed for the third family to adjust $\partial \Delta \nu_{x} / \partial J_{y}=$ $\partial \Delta \nu_{y} / \partial J_{x}$. Installation of the octupole where the beta functions are equal was not feasible because this occurs inside the final focusing quadrupole. However, a suitable location is available between this quadrupole and the neighboring final defocusing quadrupole. Figure 11 shows the exact locations of the octupoles and the beta functions at those locations. Through evaluation of multiturn tracking data, it was recognized that perfect cancellation of the three driving terms did not give best results. Higher-order contributions dominate the ADTS for larger amplitudes. Instead, the linear tune shifts were adjusted so as to limit the resulting ADTS over the entire range available within the physical aperture.

The result of the nonlinear optimization with octupoles is displayed in Figs. 12 and 13. The tune footprint of this

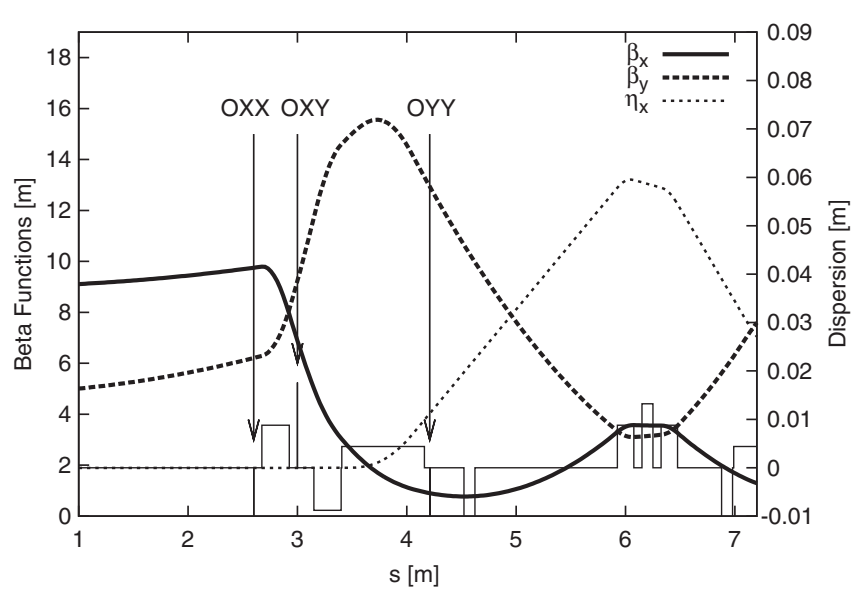

FIG. 11. Enlarged view of the optics from the long straight (left), through the matching cell and short straight to the first unit cell (right). The locations of the three octupole families as well as the beta functions $\beta_{x}, \beta_{y}$ and dispersion $\eta_{x}$ are shown. This segment is mirrored upstream of the long straight section. 


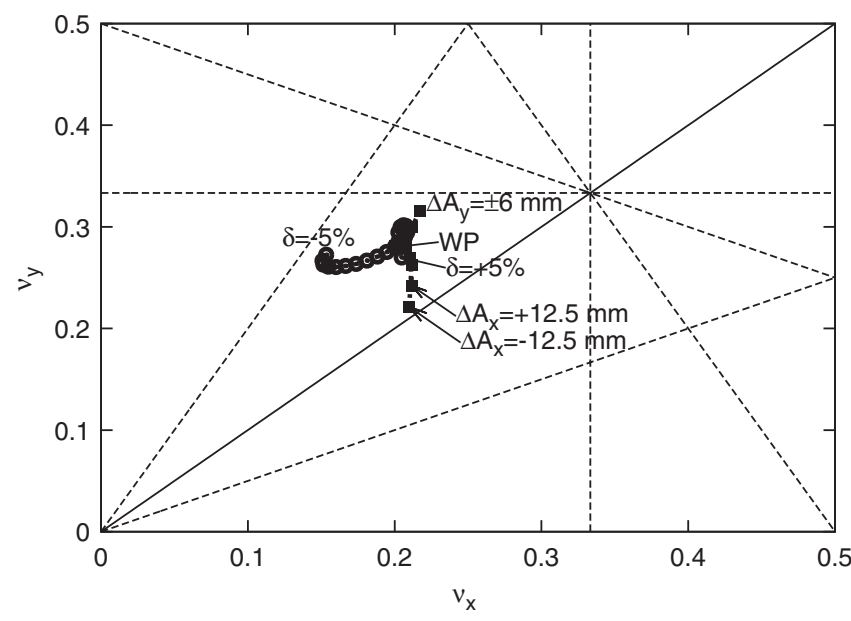

FIG. 12. Fractional tune space and tune shifts for the MAX IV $3 \mathrm{GeV}$ storage ring lattice with five sextupole families and three octupole families. The sextupole settings have been adjusted to reduce chromatic tune shifts and, hence, increase lattice MA (cf. Fig. 8). The ADTS have been suppressed with the octupoles (cf. Fig. 9). Tracking was performed with OPA and results were verified with TRACY-3.

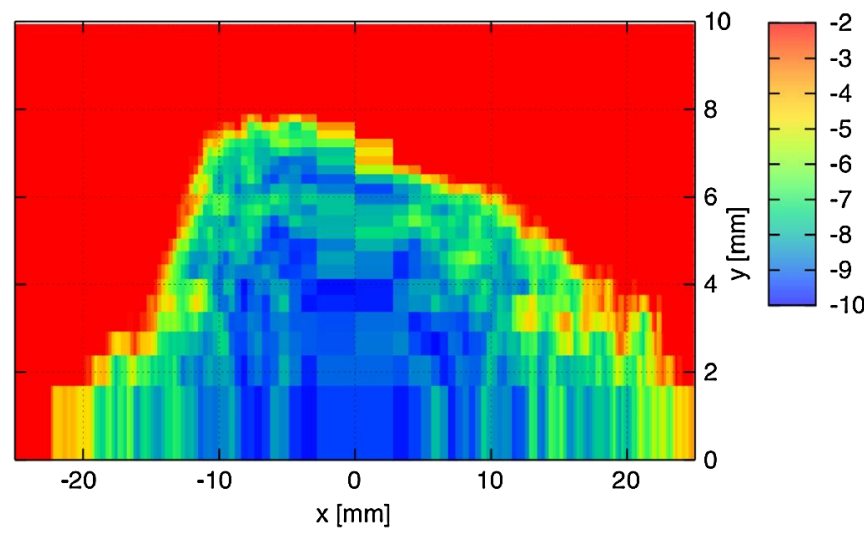

FIG. 13. Diffusion map (2000 turns) at the center of the $5 \mathrm{~m}$ long straight section for the MAX IV $3 \mathrm{GeV}$ storage ring lattice with five sextupole families and three octupole families. The scale is logarithmic in tune shift from low (blue) to high (red).

lattice has been compressed substantially. The chromatic tune footprint is still retained in a space of less than $0.07 \times 0.05$ while the ADTS has now been restricted to less than $0.02 \times 0.07$. The third-integer and second-integer resonances previously crossed for large amplitudes are no longer encountered. In fact, the only resonance encountered at all to fourth order is the vertical skew octupole resonance $4 \nu_{y}=57$ which is expected to be only weakly driven. The DA beyond the vertical acceptance has been removed and instead the horizontal DA has been enlarged. The result is a broader DA with a vertical DA that extends across the entire horizontal plane rather than being concentrated only at the center. Low betatron amplitudes see only minimal diffusion while vertical acceptance (beyond the physical acceptance in the insertion devices) has been successfully traded off for a broader horizontal acceptance. The required stay-clear aperture $[ \pm 8 \mathrm{~mm}] \times[ \pm 2.5 \mathrm{~mm}]$ shows only lowest tune shifts $(D<-9)$.

\section{Discussion}

For the MAX IV case, a couple of interesting results are worth mentioning. It is planned to correct for the influence of insertion devices both locally (matching of the achromat optics to the insertion device) and globally (restoring the working point) [3]. This can be done almost exclusively by adjusting the nondispersive matching quadrupoles at the end of the achromat. An important consequence is that this correction keeps the optical functions nearly constant within the achromat. Hence, the sextupoles do not necessarily need to be adjusted when the linear optics are matched to the insertion devices. Therefore, also the octupole settings can be left untouched when insertion device gaps change and the linear optics are corrected.

Also, because of the "wrap-up" of the chromatic tune shift around the working point, an additional installation of decapoles is not necessary. Although additional curvature of the chromatic tune footprint can be achieved by adjusting the third-order chromaticity, it is not required here: as a consequence of the $100 \mathrm{MHz}$ rf system, the linear chromaticity can be kept low which results in a lattice momentum acceptance exceeding the expected $6 \%$ maximum $\mathrm{rf}$ momentum acceptance.

It has been pointed out that folded regions of phase space are potentially unstable [49]. For amplitude excursions within the physical aperture of the MAX IV $3 \mathrm{GeV}$ storage ring, the tune shifts have been minimized so that expected tunes stay clear of the actual fold. For chromatic tune shifts, particles are expected to sample parts of the chromatic fold (cf. Fig. 12). However, in order for the fold to cause increased diffusion, resonances must be close to the actual fold and this behavior would have to show up in tracking. For this purpose DA was studied when misalignments and multipole errors [50] had been included in the tracking. Although the resulting DA is reduced somewhat by these errors, it always remains beyond the requirements and no evidence has so far been found that the fold leads to any detrimental effects. An example is shown in Fig. 14.

The required octupole strength is fairly weak: integrated strengths remain below $220 \mathrm{~m}^{-3}$. Therefore, a very compact octupole magnet $\left(L=50 \mathrm{~mm}, r_{\text {tip }}=12.5 \mathrm{~mm}\right.$, $B_{\text {tip }}<0.1 \mathrm{~T}$ ) can be used. In order to gain tuning freedom (cf. below) a slightly more generous design was chosen so that strengths up to twice the nominal values are possible. This results in very simple and inexpensive octupole magnets: the magnet length is $100 \mathrm{~mm}$ with a $150 \times 150 \mathrm{~mm}$ frame using only thin air-cooled windings.

Finally, it is foreseen to make use of the three octupole tuning knobs extensively during commissioning. As can be seen by comparing Figs. 10 and 13, the octupoles can be 


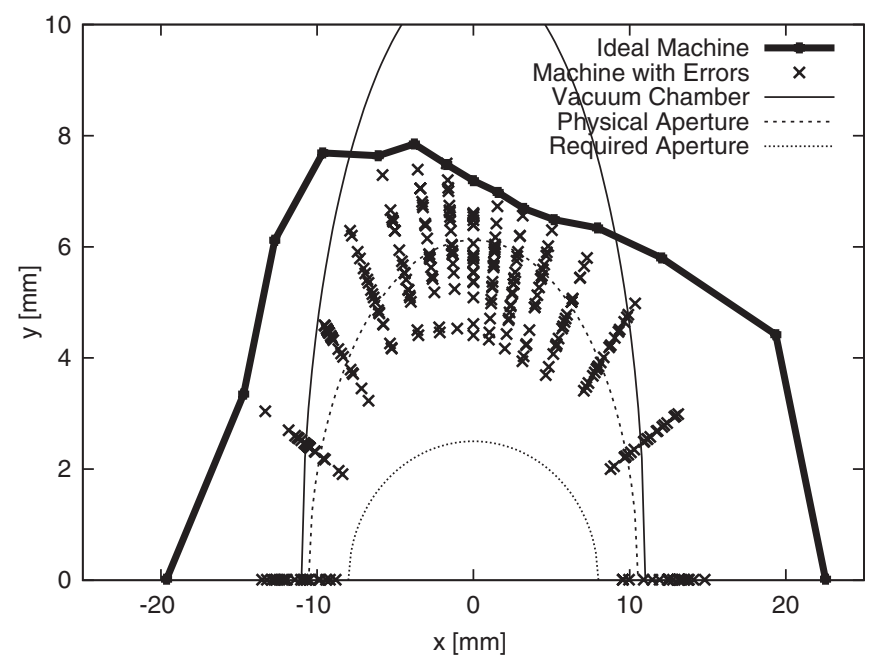

FIG. 14. On-momentum DA calculated with TRACY-3 at the center of the $5 \mathrm{~m}$ long straight section for the MAX IV $3 \mathrm{GeV}$ storage ring lattice with five sextupole families and three octupole families. The crosses show DA results for 20 seeds with realistic random error distributions (misalignments, field errors, and multipole errors) and applied orbit correction (for details of the error model, see [55]). While the DA is reduced when the errors are included, it still substantially exceeds the requirements.

used to change the ratio between available horizontal and vertical DA. The option to trade off vertical DA to increase injection efficiency is expected to be of great value during initial commissioning. Once the storage ring has been commissioned and the optics corrected for misalignments and field strength errors, the octupoles can be readjusted to the design values to maximize DA [51]. At this point it will also become known which resonances are excited in the actual machine; it could then be desirable to empirically tune the octupoles in order to adjust tune shifts away from driven resonances. Furthermore, having the octupole knobs could become useful during production to account for slight mismatches of the optics due to varying ID settings. Overall, this should result in maximizing lifetime while keeping injection efficiency high. Ultimately, the octupoles give the freedom to optimize the dynamic behavior of the storage ring for different operation scenarios.

\section{B. Example for a super-LGB lattice}

A short superconducting dipole shows a longitudinal gradient by nature, and can thus be used to build a compact hard x-ray lattice of low emittance. For example, the superbend of the ALS [25] has a roughly Gaussian longitudinal field profile $B_{y}(s)$ of $0.18 \mathrm{~m}$ FWHM and $6 \mathrm{~T}$ maximum peak field, as shown in Fig. 15.

The lattice design concept shown in Figs. 16 and 17 uses this dipole scaled to $10^{\circ}$ deflection angle at $2 \mathrm{GeV}$ as $\mathrm{CBM}$ of a triple-bend achromat structure. The achromatic arc was completed by adding $5^{\circ}$ end gradient bends for

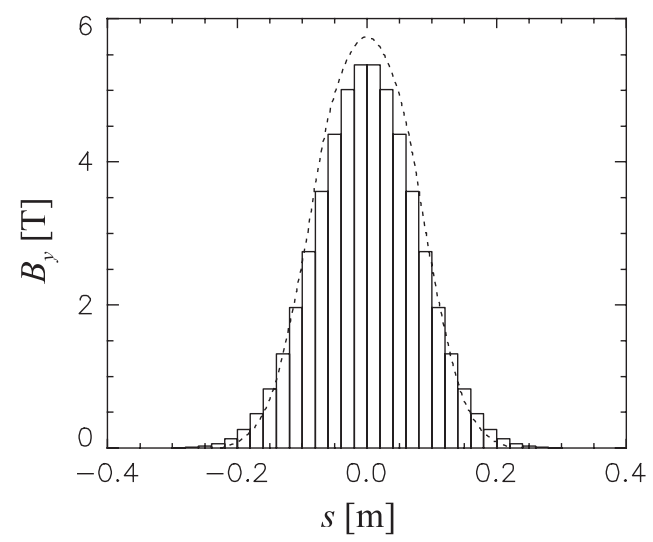

FIG. 15. Magnetic field of the ALS superbend [25] (dotted line) and approximation by a stepwise Gaussian and scaling to $10^{\circ}$ deflection angle at $2 \mathrm{GeV}$.

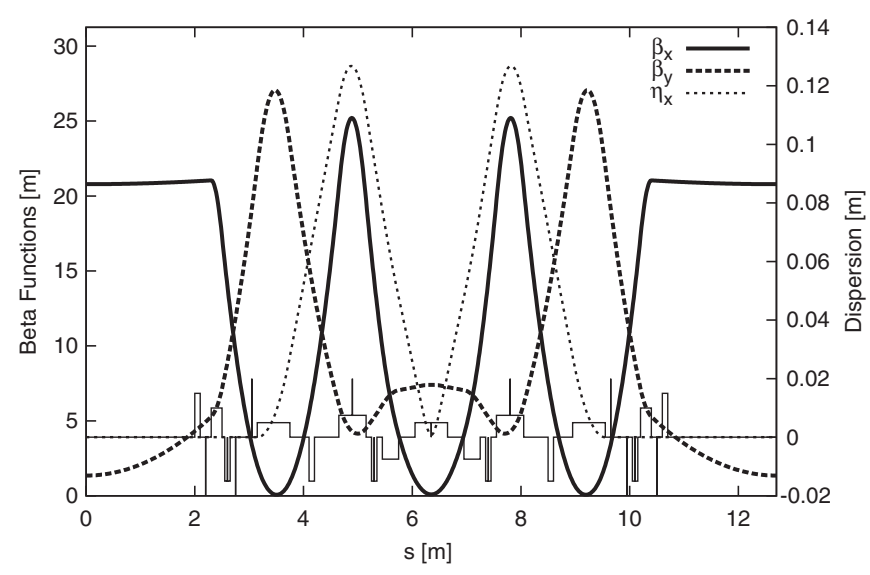

FIG. 16. Lattice cell based on a central superconducting LGB.

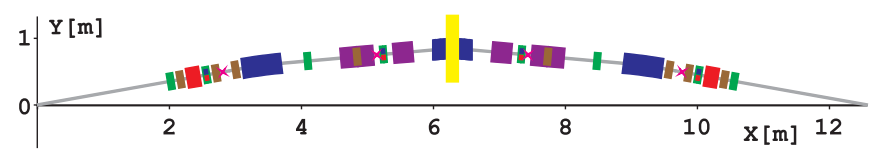

FIG. 17. Achromatic arc of the super-LGB lattice.

dispersion suppression. Combined quadrupole/sextupole magnets were used inside the achromat for dispersion matching and basic chromaticity correction. The horizontally focusing magnet also includes a weak octupole component for reduction of the second-order horizontal chromaticity. Four additional sextupole families were implemented for chromaticity tuning and DA optimization; half of the sextupoles are also equipped with dipole coils for orbit correction. Three octupole families in the dispersion-free region control the ADTS. A discrete quadrupole is required for matching the horizontal beta function to the $4 \mathrm{~m}$ long straight section. Pole face strips inside the gradient bend, as implemented in MAX III [52] for example, together with the discrete quadrupole family would allow moving the working point in the tune diagram. 18 of 
these cells would form a ring of $228.6 \mathrm{~m}$ circumference for 18 hard x-ray beam lines and, taking into account space required for injection and $\mathrm{rf}$, about 15 soft $\mathrm{x}$-ray undulator beam lines.

The parameters of this lattice are given in Table I. The dispersion in the center of the super-LGB was set exactly to the TME condition [i.e. $d=1$, cf. Eq. (6)], whereas the horizontal beta function was relaxed $(b=3.7)$ to limit the cell phase advance. Nevertheless, the emittance is lower than the TME of a homogenous $10^{\circ}$ dipole $(0.67 \mathrm{~nm}$ at $2 \mathrm{GeV}$ ). The homogenous EBMs of the $5^{\circ}$ angle provide about the same emittance as the super-LGB CBM.

The chromaticities are large, but most of it is corrected locally, where it is created, which avoids momentumdependent beta beats and thus allows large MA. With an elliptic vacuum chamber of $30 \mathrm{~mm}$ full width and $20 \mathrm{~mm}$ full height as it is used in the SLS booster synchrotron [33], the maximum dispersion of $0.13 \mathrm{~m}$ allows a physical MA of $\pm 5.7 \%$. The optimization of DA and dynamic MA was done in a way similar to the MAX IV design. The octupoles were set to create a fold of the frequency map just outside the vacuum chamber, as shown in Fig. 18: this minimizes the amplitude-dependent tune footprint while providing maximum tune spread in the beam core region for Landau damping of transverse instabilities [53].

The resulting DA of the ideal lattice does not significantly reduce the aperture defined by the vacuum chamber over the full range of energies as shown in Fig. 19. Assuming $500 \mathrm{~mA}$ total current, $1 \%$ emittance coupling, and a $100 \mathrm{MHz}$ rf system of $800 \mathrm{kV}$ peak voltage results in $7.5 \mathrm{hrs}$ of Touschek lifetime.

Orbit amplification factors were found to be 60 horizontal, 33 vertical for single element misalignments of $50 \mu \mathrm{m}$ and $50 \mu \mathrm{rad} \mathrm{rms}$; however, in reality the magnets of one arc would be combined in three or even one combined girder/ yoke resulting in lower relative misalignments.

The small vacuum chamber allows high multipole gradients at moderate pole-tip fields. In this example, the poletip field in the gradient bend amounts to $1.35 \mathrm{~T}$, in the quadrupole/sextupoles to $<0.5 \mathrm{~T}$, in the sextupoles to $0.25 \mathrm{~T}$, and to $<0.1 \mathrm{~T}$ in the octupoles.

The draft design presented here does not yet prove feasibility, and the radiation available from the machine

TABLE I. Parameters of the super-LGB test lattice.

\begin{tabular}{lc}
\hline \hline Circumference $[\mathrm{m}]$ & 228.6 \\
\hline Working point & $26.28 / 8.71$ \\
Chromaticities & $-160 /-53$ \\
Momentum compaction factor & $1.7 \times 10^{-4}$ \\
Horizontal damping partition $J_{x}$ & 1.04 \\
Energy [GeV] & 2.0 \\
Emittance [nm rad] & 0.55 \\
rms energy spread & $1.37 \times 10^{-3}$ \\
Energy loss per turn $[\mathrm{keV}]$ & 554 \\
\hline \hline
\end{tabular}

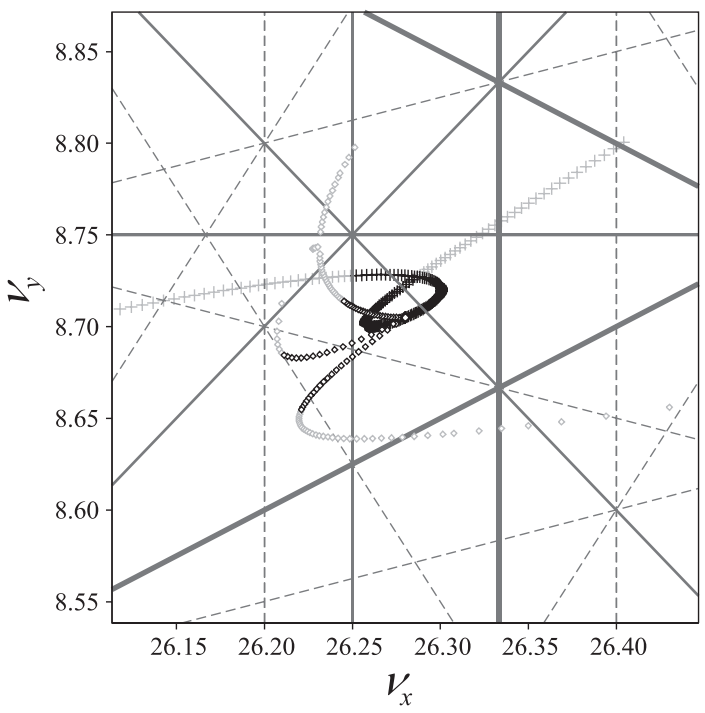

FIG. 18. Tune footprint of the super-LGB lattice. Crosses mark the chromatic tune shift for energy variation from $-7.0 \%$ (left) to $+7.5 \%$ (right). The three diamond curves mark the tune shifts with vertical (upper), horizontal (lower), and combined amplitude (middle). Only the points in black are inside the vacuum chamber. Resonances of third (thick solid), fourth (thin solid), and fifth order (thin dashed) are shown.

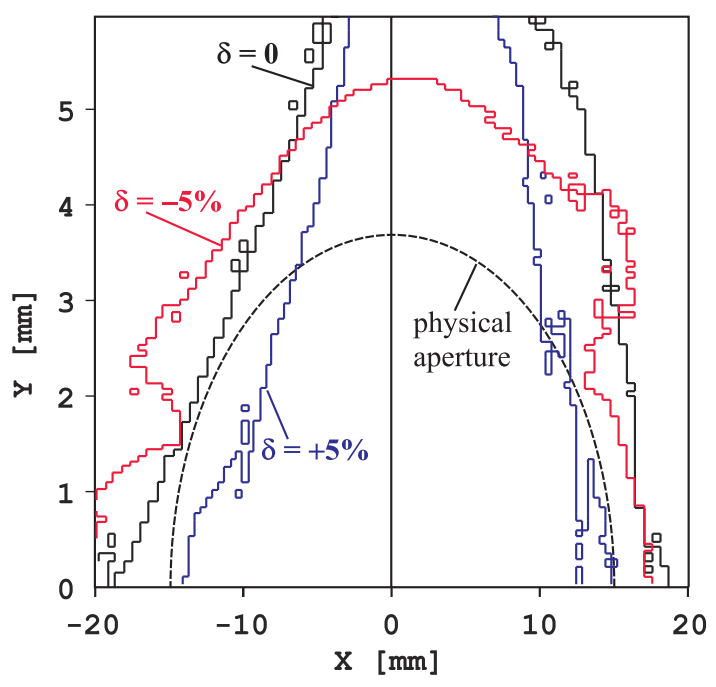

FIG. 19. Dynamic apertures of the ideal super-LGB lattice.

would probably not meet user requirements, but as a mere case study it does indeed show how a compact hard $\mathrm{x}$-ray machine of high brightness could be realized by exploiting the longitudinal field variation of a superconducting dipole magnet.

\section{Momentum acceptance of a compact light source}

Compact light sources are usually designed for high flux rather than for highest brightness, and therefore have fewer lattice cells, larger deflection angles per dipole, and hence larger dispersion. Achieving sufficient DA is usually less 


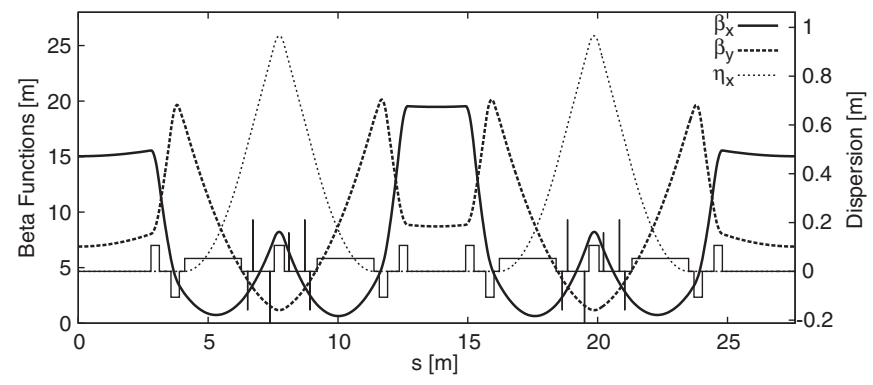

FIG. 20. Optical function for the ANKA light source. Octupoles, shown as thin, long lines in the magnet plot, were added irrespective of geometric conditions of the real machine.

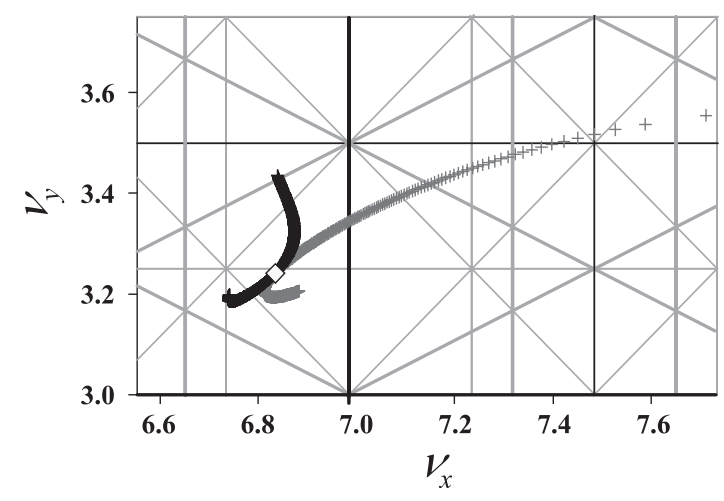

FIG. 21. Chromatic tune shifts of the ANKA lattice without (gray) and with (black) two families of octupoles for an energy range of $\pm 3 \%$. The diamond marks the working point.

challenging and therefore two sextupole families are often considered sufficient. Nevertheless, second-order chromaticity may restrict the MA. It can, however, be corrected in a straightforward way using two families of octupoles in the dispersive region. As an example, we have chosen the lattice of the ANKA light source [54]. Figure 20 displays the optical functions for a working point at 6.85/3.22 and Fig. 21 shows the chromatic tune footprint with and without octupoles for linear chromaticities of $+5 /+5$. Because of the large dispersion, the octupoles are well feasible: With a magnet gap of $70 \mathrm{~mm}$ and a length of $150 \mathrm{~mm}$, the maximum pole-tip field would be $0.17 \mathrm{~T}$ at $2.5 \mathrm{GeV}$.

\section{CONCLUSIONS}

Longitudinal gradient bending magnets (LGB) have not yet been exploited for low-emittance lattices. However, application appears promising only for high-field (superconducting) LGBs operated slightly above TME conditions in order to limit the cell length and make use of hard $\mathrm{x}$ rays from the central high-field region. In general, LGBs used for ultralow emittance lead to rather long lattice cells and thus appear inferior to multibend achromat schemes.
Octupoles provide highly efficient knobs for controlling ADTS. As such they are a powerful tool for design, commissioning, and operation of an ultralow-emittance light source. In ultralow-emittance design, where the secondorder optimization of sextupoles is exhausted, octupoles actually become essential for realization of sufficient DA. This is exemplified in the ultralow-emittance design of the MAX IV $3 \mathrm{GeV}$ storage ring lattice where octupoles have become an integral part of the lattice design.

Control of second-order chromaticity with octupoles or control of third-order chromaticity with decapoles is, although in principle possible, less efficient due to the rather low dispersion encountered in ultralow-emittance lattices where very many dipoles with small bending angles are used. In compact light sources however, where dispersion is often larger, application of octupoles or decapoles to correct higher-order chromatic effects is straightforward. Comparably weak octupoles can already lead to a substantial reduction of tune footprint.

The application of octupoles and decapoles as presented here neglects the resonance driving terms, assuming that the multipoles are "weak"- similar to the situation that existed for sextupoles in relaxed lattices, where only chromaticity correction had to be considered. Straightforward continuation of this work would lead to making use of the complete linear system of first-order octupole and decapole Hamiltonians, as is common practice today for sextupoles.

\section{APPENDIX A: EMITTANCE AND PHASE ADVANCE}

For a homogenous bending magnet, the radiation integral $I_{5}$ from Eq. (2) is given by $I_{5}=h^{3}\langle\mathcal{H}\rangle_{\text {mag }}=$ $h^{3} \int_{L} \mathcal{H}(s) d s$, i.e., by averaging the dispersion amplitude from Eq. (3) over the bending magnets [9]. The matching conditions for obtaining the minimum emittance in the two cases of the center bend (CBM) and the end bend (EBM) are obtained by minimizing

$$
\begin{aligned}
& \mathrm{CBM}: \partial \mathcal{H} / \partial \beta_{x o} \stackrel{!}{=} 0 \quad \partial \mathcal{H} / \partial \eta_{o} \stackrel{!}{=} 0 \\
& \text { EBM: } \partial \mathcal{H} / \partial \beta_{x o} \stackrel{!}{=} 0 \quad \partial \mathcal{H} / \partial l \stackrel{!}{=} 0,
\end{aligned}
$$

where $\beta_{x o}$ is the beta function at the focus inside the magnet, $\eta_{o}$ is the dispersion at the focus (CBM), and $l$ is the location of the focus from the entrance edge (EBM).

Assuming a small deflection angle $\Phi=h L \ll 1$ and negligible gradient, the transfer matrix for $\left(x, x^{\prime} \mid \delta\right)$ is approximately given by

$$
\left(\begin{array}{ll|c}
1 & s & h s^{2} / 2 \\
0 & 1 & h s
\end{array}\right)
$$

Application to $\beta_{x}(s)$ and $\eta(s)$ with initial conditions $\beta_{x o}$, $\eta_{o}$ at the center focus gives for the CBM 
$\langle\mathcal{H}\rangle_{\mathrm{mag}}=\frac{h^{2}}{\beta_{\text {xo }}}\left[\frac{(L / 2)^{4}}{20}+\frac{(L / 2)^{2}}{3}\left(\beta_{x o}^{2}-\frac{\eta_{o}}{h}\right)+\left(\frac{\eta_{o}}{h}\right)^{2}\right]$

For the EBM we get with $\beta_{x o}$ at the focus in distance $l$ from the entrance (EBM) edge:

$$
\langle\mathcal{H}\rangle_{\mathrm{mag}}=\frac{h^{2}}{\beta_{\text {xo }}}\left[\frac{L^{4}}{20}+\frac{L^{2}}{3}\left(\beta_{x o}^{2}+l^{2}\right)-\frac{l L^{3}}{4}\right] .
$$

Minimization yields the matching conditions from Eq. (5) and inserting these results,

$$
\langle\mathcal{H}\rangle_{\operatorname{mag}}^{\min }=\frac{h^{2} L^{3}}{12 \sqrt{15}} F^{\min }
$$

with $F_{\mathrm{CBM}}^{\min }=1$ and $F_{\mathrm{EBM}}^{\min }=3$ [13].

Introducing Eq. (A6) and the parameters for deviations from the TME conditions from Eq. (6) into Eqs. (A4) and (A5) results in

CBM: $F=\left(9+4 b^{2}-10 d+5 d^{2}\right) /(8 b)$

EBM: $F=\left(48+3 b^{2}-240 \lambda+320 \lambda^{2}\right) /(2 b)$

which can nicely be written as ellipse equations, cf. Eq. (7).

In order to calculate the cell phase advance, we consider the transfer matrix $R$ from the focus " 0 " to the point " $\mathrm{P}$ " $(\mathrm{CBM})$, respectively " $\mathrm{M}$ " (EBM), where we require $\alpha_{x P / M}=\eta_{P / M}^{\prime}=0$ in order to build a periodic cell (CBM), respectively a mirror cell (EBM) (cf. Fig. 1). This gives two matching conditions for the matrix $R$ :

$$
\begin{aligned}
& 0 \stackrel{!}{=} \alpha_{x P / M}=-r_{11} r_{21} \beta_{x o}-r_{12} r_{22} \frac{1}{\beta_{x o}} \\
& 0 \stackrel{!}{=} \eta_{P / M}^{\prime}=r_{21} \hat{\eta}_{0}+r_{22} \hat{\eta}_{0}^{\prime} .
\end{aligned}
$$

Here we took into account the bending magnet's dispersion production [i.e. the right column of its matrix from Eq. (A3)] by introducing $\hat{\eta}_{0}, \hat{\eta}_{0}^{\prime}$, which is the back transformation of dispersion from the bending magnet exit edge to the location of the focus:

$$
\begin{array}{ll}
\text { CBM: } \hat{\eta}_{o}=\eta_{o}-h L^{2} / 8 & \hat{\eta}_{o}^{\prime}=h L / 2 \\
\text { EBM: } \hat{\eta}_{o}=h L^{2}(l-L / 2) & \hat{\eta}_{o}^{\prime}=h L .
\end{array}
$$

In the CBM case the matrix of the periodic cell is given by

$$
M=T R^{-1} T R \quad \text { with } T=\left(\begin{array}{cc}
1 & 0 \\
0 & -1
\end{array}\right),
$$

the mirror matrix to be applied when changing from forward to backward calculation and vice versa. In the EBM case, the same equation applies to a "pseudoperiodic" cell from point " 0 " to its mirror image on the other side of point "M", which is all we can consider in the EMB case: since the dispersion constraint does not exist for matching to point " $S$ ", the matrix for the left side of the cell [cf. Fig. 1 (lower)] is not determined. The cell phase advance is obtained from the trace of the periodic or pseudoperiodic matrix:

$$
\cos \mu=\frac{m_{11}+m_{22}}{2}=r_{11} r_{22}+r_{12} r_{21} .
$$

Using symplecticity, $|R|=r_{11} r_{22}-r_{12} r_{21}=1$, only one of the products, $r_{11} r_{22}$ or $r_{12} r_{21}$, remains to be determined. Thus, although the matrix $R$ itself is not determined, the cell phase advance can be calculated from the three constraints given (the two matching conditions and symplecticity):

$$
\tan \frac{\mu}{2}=\sqrt{\frac{1-\cos \mu}{1+\cos \mu}}=\frac{\hat{\eta}_{o}^{\prime}}{\hat{\eta}_{o}} \beta_{x o} .
$$

Inserting the back transformed dispersion from Eq. (A9), the minimum emittance values from Eq. (5) and the parameters $b, d, \lambda$ from Eq. (6), we obtain the cell phase advance from Eq. (8).

\section{APPENDIX B: EFFECTIVE EMITTANCE}

In a dispersive region, the effective emittance is obtained from a convolution of the 2D Gaussian distribution of betatron amplitudes with the 1D Gaussian distribution of momenta.

In normalized phase space, using coordinates

$$
\chi=\frac{x}{\sqrt{\beta}} \quad \chi^{\prime}=\frac{\alpha x+\beta x^{\prime}}{\sqrt{\beta}},
$$

the $1-\sigma$ beam ellipse of the 2D betatron amplitude distribution is transformed into a circle with radius $\sqrt{\epsilon_{o}}$, and the $1 \mathrm{D}$ distribution of momenta will extend along a line with a distribution of standard deviation $\sigma_{\eta}=\sqrt{\mathcal{H}} \sigma_{\delta}$, with $\mathcal{H}$ from Eq. (3) and $\sigma_{\delta}$ the natural energy spread.

The standard deviation of the resulting beam distribution parallel to this line is given by convolution, whereas the standard deviation perpendicular to this line remains unchanged:

$$
\sigma_{\|}^{2}=\epsilon_{o}+\mathcal{H} \sigma_{\delta}^{2} \quad \sigma_{\perp}^{2}=\epsilon_{o} .
$$

The effective emittance is given as the product of both:

$$
\epsilon_{\mathrm{eff}}=\sigma_{\|} \sigma_{\perp}=\sqrt{\epsilon_{o}^{2}+\epsilon_{o} \mathcal{H} \sigma_{\delta}^{2}} .
$$

\section{APPENDIX C: OCTUPOLE HAMILTONIAN}

The octupole Hamiltonian can be expanded for small deviations from design momentum $\delta=\Delta p / p$ and written as

$$
H_{4}=\frac{b_{4}}{4}\left[1-\delta+\delta^{2}+\mathcal{O}\left(\delta^{3}\right)\right]\left(x^{4}-6 x^{2} y^{2}+y^{4}\right),
$$

where $b_{4}$ is the normalized integrated strength of a regular octupole. In most lattices many octupoles will be 
distributed throughout the lattice. Because of linear superposition, we may add contributions to $H_{4}$ from individual octupoles in the lattice. In an ideal flat machine with perfect magnets betatron motion can be expressed as

$$
x=x_{\beta}+\eta \delta, \quad y=y_{\beta},
$$

where $x_{\beta}$ and $y_{\beta}$ describe horizontal and vertical betatron motion around the design orbit and $\eta$ is the horizontal dispersion. Inserting this into Eq. (C1), the Hamiltonian can be expanded to second order in momentum

$$
\begin{aligned}
H_{4}= & \frac{b_{4}}{4}\left\{(\text { oct })+\delta[-(\text { oct })+4 \eta(\text { sext })]+\delta^{2}[(\text { oct })\right. \\
& \left.\left.-4 \eta(\text { sext })+6 \eta^{2}(\text { quad })\right]\right\}+\mathcal{O}\left(\delta^{3}\right)
\end{aligned}
$$

where the three following terms have been recognized:

$$
\begin{gathered}
\text { oct }=x_{\beta}^{4}-6 x_{\beta}^{2} y^{2}+y^{4}, \\
\text { sext }=x_{\beta}^{3}-3 x_{\beta} y^{2}, \\
\text { quad }=x_{\beta}^{2}-y^{2}
\end{gathered}
$$

At this point betatron motion can be expressed in the "resonance basis" [38]:

$$
h_{x, y^{ \pm}}=\sqrt{2 J_{x, y} \beta_{x, y}} e^{ \pm i \phi_{x, y},}
$$

where $J_{x, y}$ is the amplitude and $\phi_{x, y}$ the phase of the betatron motion. This can be inserted into the Hamiltonian [Eq. (C3)] by recalling that

$$
\begin{aligned}
& x_{\beta}=\frac{1}{2}\left(h_{x^{+}}+h_{x^{-}}\right), \\
& y_{\beta}=\frac{1}{2}\left(h_{y^{+}}+h_{y^{-}}\right) .
\end{aligned}
$$

It is convenient to then express the octupole Hamiltonian in the resonance basis:

$$
\begin{aligned}
H_{4}= & \sum h_{j k l m n}=\sum \alpha_{j k l m n} h_{x^{+}}^{j} h_{x^{-}}^{k} h_{y^{+}}^{l} h_{y^{-}}^{m} \delta^{n} \\
= & \sum \alpha_{j k l m n}\left(2 J_{x} \beta_{x}\right)^{(j+k) / 2} \\
& \times\left(2 J_{y} \beta_{y}\right)^{(l+m) / 2} e^{i\left[(j-k) \phi_{x}+(l-m) \phi_{y}\right]} \delta^{n} .
\end{aligned}
$$

Summands for which the sum of indices $(j+k+l+m+$ $n)=4$ are first-order octupole terms; there are a total of 36 such terms in the octupole Hamiltonian. Those containing phases, i.e. terms where either $(j-k) \neq 0$ or $(l-m) \neq 0$ drive betatron resonances: $Q_{x}, 2 Q_{x}, 2 Q_{y}, 3 Q_{x}, Q_{x} \pm 2 Q_{y}$, $2 Q_{x} \pm 2 Q_{y}, 4 Q_{x}, 4 Q_{y}$. For the octupole Hamiltonian there exist 30 such terms for momentum deviations up to second order. One example is

$$
h_{02200}=h_{20020}^{*}=-\frac{3 b_{4}}{32} \beta_{x} \beta_{y}\left(2 J_{x}\right)\left(2 J_{y}\right) e^{-i\left(2 \phi_{x}-2 \phi_{y}\right)}
$$

which obviously drives the $2 Q_{x}-2 Q_{y}$ resonance.

There are however also five terms (to second order in momentum deviation) in the octupole Hamiltonian that do not contain phases. They are

$$
\begin{gathered}
h_{22000}=\frac{3}{8} b_{4} \beta_{x}^{2} J_{x}^{2}, \\
h_{11110}=-3 b_{4} \beta_{x} \beta_{y} J_{x} J_{y}, \\
h_{00220}=\frac{3}{8} b_{4} \beta_{y}^{2} J_{y}^{2}, \\
h_{11002}=\frac{3}{2} b_{4} \beta_{x} J_{x} \eta^{2} \delta^{2}, \\
h_{00112}=-\frac{3}{2} b_{4} \beta_{y} J_{y} \eta^{2} \delta^{2} .
\end{gathered}
$$

These five terms are tune shifts, which is easily recognized if one recalls that $J$ and $2 \pi \nu$ are conjugate variables, that is,

$$
\Delta \nu_{x, y}=\frac{1}{2 \pi} \frac{\partial H}{\partial J_{x, y}} .
$$

The first three terms (second-order amplitude dependence) express ADTS:

$$
\begin{aligned}
\frac{\partial \Delta \nu_{x, y}}{\partial J_{x, y}} & =\frac{1}{2 \pi} \frac{\partial^{2} H_{4}}{\partial J_{x, y}^{2}} \\
& =\frac{1}{2 \pi} \frac{\partial^{2}}{\partial J_{x, y}^{2}}\left(h_{22000}+h_{00220}+h_{11110}\right), \\
\rightarrow \frac{\partial \Delta \nu_{x}}{\partial J_{x}} & =\frac{3}{8 \pi} b_{4} \beta_{x}^{2} \\
\rightarrow \frac{\partial \Delta \nu_{y}}{\partial J_{y}} & =\frac{3}{8 \pi} b_{4} \beta_{y}^{2}, \\
\rightarrow \frac{\partial \Delta \nu_{x}}{\partial J_{y}} & =\frac{\partial \Delta \nu_{y}}{\partial J_{x}}=-\frac{3}{4 \pi} b_{4} \beta_{x} \beta_{y} .
\end{aligned}
$$

The two remaining terms show only linear dependence on amplitude. They are therefore chromatic tune shifts, in fact they express the quadratic chromaticity:

$$
\begin{aligned}
\xi_{x, y}^{(2)} & =\frac{1}{2} \frac{\partial^{2} \Delta \nu_{x, y}}{\partial \delta^{2}}=\frac{1}{4 \pi} \frac{\partial^{2}}{\partial \delta^{2}} \frac{\partial}{\partial J_{x, y}}\left(h_{11002}+h_{00112}\right) \\
& =\frac{6 b_{4}}{16 \pi} \eta^{2} \frac{\partial^{2}}{\partial \delta^{2}} \delta^{2} \frac{\partial}{\partial J_{x, y}}\left(\beta_{x} J_{x}-\beta_{y} J_{y}\right) \\
\rightarrow \xi_{x, y}^{(2)} & = \pm \frac{3 b_{4}}{4 \pi} \eta^{2} \beta_{x, y} .
\end{aligned}
$$




\section{APPENDIX D: DECAPOLE HAMILTONIAN}

The decapole Hamiltonian can be treated analogously to the octupole Hamiltonian (cf. Appendix C). For small deviations from design momentum $\delta=\Delta p / p$ it is expanded to

$H_{5}=\frac{b_{5}}{5}\left[1-\delta+\delta^{2}-\delta^{3}+\mathcal{O}\left(\delta^{4}\right)\right]\left(x^{5}-10 x^{3} y^{2}+5 x y^{4}\right)$,

where $b_{5}$ is the normalized integrated strength of a regular decapole. Betatron motion in an ideal flat machine with perfect magnets is again expressed as in Eq. (C2) which leads to the following Hamiltonian:

$$
\begin{aligned}
H_{5}= & \frac{b_{5}}{5}\{(\mathrm{dec})+\delta[-(\mathrm{dec})+5 \eta(\mathrm{oct})] \\
& +\delta^{2}\left[(\mathrm{dec})-5 \eta(\mathrm{oct})+10 \eta^{2}(\mathrm{sext})\right] \\
& \left.+\delta^{3}\left[-(\mathrm{dec})+5 \eta(\mathrm{oct})-10 \eta^{2}(\mathrm{sext})+10 \eta^{3}(\text { quad })\right]\right\} \\
& +\mathcal{O}\left(\delta^{4}\right),
\end{aligned}
$$

where again the definitions in Eqs. (C4)-(C6) have been made use of in addition to

$$
\operatorname{dec}=x_{\beta}^{5}-10 x_{\beta}^{3} y^{2}+5 x_{\beta} y^{4} .
$$

The Hamiltonian can then again be expressed in the resonance basis [Eqs. (C7)-(C10)]. There are in total 65 decapolar terms in the Hamiltonian (63 up to third order in momentum) of which 60 carry phases and hence drive resonances. The remaining five terms are tune shifts which shall be examined here. Keeping in mind Eq. (C17), the third-order chromaticity is derived by inspecting terms $h_{j k l m n}$ for which $n=3$ :

$$
\begin{aligned}
\xi_{x, y}^{(3)} & =\frac{1}{6} \frac{\partial^{3} \Delta \nu_{x, y}}{\partial \delta^{3}}=\frac{1}{12 \pi} \frac{\partial^{3}}{\partial \delta^{3}} \frac{\partial}{\partial J_{x, y}}\left(h_{11003}+h_{00113}\right) \\
& =\frac{b_{5}}{6 \pi} \eta^{3} \frac{\partial^{3}}{\partial \delta^{3}} \delta^{3} \frac{\partial}{\partial J_{x, y}}\left(\beta_{x} J_{x}-\beta_{y} J_{y}\right) \\
\rightarrow \xi_{x, y}^{(3)} & = \pm \frac{b_{5}}{\pi} \eta^{3} \beta_{x, y} .
\end{aligned}
$$

The remaining three tune shifts in the decapole Hamiltonian are

$$
\begin{gathered}
h_{22001}=\frac{3}{2} b_{5} \beta_{x}^{2} J_{x}^{2} \eta \delta, \\
h_{00221}=\frac{3}{2} b_{5} \beta_{x}^{2} J_{x}^{2} \eta \delta, \\
h_{11111}=-6 b_{5} \beta_{x} \beta_{y} J_{x} J_{y} \eta \delta .
\end{gathered}
$$

These are essentially ADTS for off-momentum particles because, as one would expect, for such particles a decapole feeds down as an octupole. The ADTS are expressed analogously to Eqs. (C18)-(C20):

$$
\begin{aligned}
\frac{\partial \Delta \nu_{x, y}}{\partial J_{x, y}} & =\frac{1}{2 \pi} \frac{\partial^{2} H_{5}}{\partial J_{x, y}^{2}} \\
& =\frac{1}{2 \pi} \frac{\partial^{2}}{\partial J_{x, y}^{2}}\left(h_{22001}+h_{00221}+h_{11111}\right), \\
\rightarrow \frac{\partial \Delta \nu_{x}}{\partial J_{x}} & =\frac{3}{2 \pi} b_{5} \beta_{x}^{2} \eta \delta, \\
\rightarrow \frac{\partial \Delta \nu_{y}}{\partial J_{y}} & =\frac{3}{2 \pi} b_{5} \beta_{y}^{2} \eta \delta, \\
\rightarrow & \frac{\partial \Delta \nu_{x}}{\partial J_{y}}=\frac{\partial \Delta \nu_{y}}{\partial J_{x}}=-\frac{3}{\pi} b_{5} \beta_{x} \beta_{y} \eta \delta
\end{aligned}
$$

containing the explicit dependence on momentum deviation.

[1] K. Balewski, W. Brefeld, W. Decking, H. Franz, R. Röhlsberger, and E. Weckert, PETRA III Technical Design Report, 2004, http://petra3.desy.de/general/tdr/.

[2] NSLS-II Preliminary Design Report, 2007, http:// www.bnl.gov/nsls2/project/PDR/.

[3] S. C. Leemann, A. Andersson, M. Eriksson, L.-J. Lindgren, E. Wallén, J. Bengtsson, and A. Streun, Phys. Rev. ST Accel. Beams 12, 120701 (2009).

[4] M. Bei, M. Borland, Y. Cai, P. Elleaume, R. Gerig, K. Harkay, L. Emery, A. Hutton, R. Hettel, R. Nagaoka, and D. Robin, C. Steier, Nucl. Instrum. Methods Phys. Res., Sect. A 622, 518 (2010).

[5] S. Krinsky and R. Hettel, Summary Report: Working Group on Storage Ring Sources, Future Light Source Workshop 2010, SLAC, 2010, https://slacportal.slac .stanford.edu/sites/ad_public/events/FLS2010/Lists/FLS $\% 202010 \% 20$ Workshop\%20Program/Attachments/ 7/Summary_WG2-StorageRings.pdf.

[6] M. Borland, Overview of Ring-Based X-ray Sources, Future Light Source Workshop 2010, SLAC, 2010, https://slacportal.slac.stanford.edu/sites/ad_public/events/ FLS2010/Lists/FLS\%202010\%20Workshop\%20Program/ Attachments/42/RingOverview10.pdf.

[7] Y. Nosochkov, Lattice Design and Performance for PEP-X Light Source, Future Light Source Workshop 2010, SLAC, 2010, https://slacportal.slac.stanford.edu/ sites/ad_public/events/FLS2010/Lists/WorkingGroup2/ Attachments/20/PEPX_lattice.pdf.

[8] T. Watanabe, SPring-8 Upgrade Plan, Future Light Source Workshop 2010, SLAC, 2010, https://slacportal .slac.stanford.edu/sites/ad_public/events/FLS2010/Lists/ WorkingGroup2/Attachments/21/SPring-8\%20upgrade\% 20-\%20Watanabe.pdf.

[9] M. Sands, Internal Report No. SLAC-121, Stanford, 1970.

[10] Other authors generally use the term TME magnet for a magnet with symmetric optical functions. We refer to this type of magnet as a CBM. In our definition, a TME magnet is a magnet providing exactly the minimum emittance theoretically obtainable. 
[11] D. Trbojevic and E. Courant, in Proceedings of the Fourth European Particle Accelerator Conference EPAC 94, London, England (World Scientific, River Edge, NJ, 1994), p. 1000.

[12] D. Einfeld and M. Plesko, Nucl. Instrum. Methods Phys. Res., Sect. A 335, 402 (1993).

[13] L. C. Teng, ANL Internal Report No. LS-17, 1985.

[14] J.P. Potier and L. Rivkin, in Proceedings of the 1997 Particle Accelerator Conference, Vancouver, BC (IEEE, New York, 1998), pp. 476-478.

[15] P. Emma and T. Raubenheimer, Phys. Rev. ST Accel. Beams 4, 021001 (2001).

[16] W. Joho and L. Rivkin (unpublished).

[17] M. Bassetti, C. J. Bocchetta, and A. Wrulich, Internal Report No. ST/M-87/10, Trieste, 1987.

[18] A. Jackson et al., in Proceedings of EPAC 1988, Rome, Italy (Institute of Physics Publishing, Bristol and Philadelphia, 1988), pp. 359-361.

[19] M. Muñoz and D. Einfeld, in Proceedings of the 21st Particle Accelerator Conference, Knoxville, 2005 (IEEE, Piscataway, NJ, 2005), pp. 3777-3779.

[20] L. Farvacque et al., in Proceedings of the Fourth European Particle Accelerator Conference EPAC 94, London, England (Ref. [11]), pp. 612-614.

[21] M.P. Level et al., in Proceedings of EPAC 1996, Sitges, Spain, 1996 (Institute of Physics Publishing, Bristol, 1996), pp. 670-672.

[22] J. Guo and T. Raubenheimer, in Proceedings of the 8th European Particle Accelerator Conference, Paris, 2002 (EPS-IGA and CERN, Geneva, 2002), p. 1136.

[23] R. Nagaoka and A. Wrulich, Nucl. Instrum. Methods Phys. Res., Sect. A 575, 292 (2007).

[24] A. Streun, PSI Internal Report No. SLS-TME-TA-20060297, 2004.

[25] D. Robin et al., in Proceedings of the Particle Accelerator Conference, Chicago, IL, 2001 (IEEE, New York, 2001), pp. 2632-2634.

[26] W. Joho, P. Marchand, L. Rivkin, and A. Streun, in Proceedings of the Fourth European Particle Accelerator Conference EPAC 94, London, England (Ref. [11]), pp. 627-629.

[27] D. Einfeld, J. Schaper, and M. Plesko, in Proceedings of the Particle Accelerator Conference, Dallas, TX, 1995 (IEEE, New York, 1995), pp. 177-179.

[28] D. Kaltchev, R. V. Servranckx, M. K. Crddock, and W. Joho, in Proceedings of the Particle Accelerator Conference, Dallas, TX, 1995 (Ref. [27]), pp. 28232825.

[29] H. Tarawneh, M. Eriksson, L. J. Lindgren, and B. Anderberg, Nucl. Instrum. Methods Phys. Res., Sect. A 508, 480 (2003).

[30] M. Eriksson, L.-J. Lindgren, M. Sjöström, E. Wallén, L. Rivkin, and A. Streun, Nucl. Instrum. Methods Phys. Res., Sect. A 587, 221 (2008).

[31] W.D. Klotz and G. Mülhaupt, in Proceedings of the Workshop on 4th Generation Light Sources, Stanford, CA, 1992.

[32] G. Mülhaupt, ESRF Grenoble Internal Report No. ESRF/ MACH-INJ/94-13, 1994.

[33] W. Joho, M. Muñoz, and A. Streun, Nucl. Instrum. Methods Phys. Res., Sect. A 562, 1 (2006).
[34] G. Benedetti et al., in Proceedings of the 11th European Particle Accelerator Conference, Genoa, 2008 (EPS-AG, Genoa, Italy, 2008), pp. 2148-2150.

[35] M. Georgsson, N. Hauge, and S. P. Møller, in Proceedings of EPAC 2004, Lucerne, Switzerland, 2004 (EPS-AG and CERN, Geneva, 2004), pp. 2317-2319.

[36] The SLS booster provides $\epsilon=10 \mathrm{~nm} \mathrm{rad}$ at $2.4 \mathrm{GeV}$ [33].

[37] J. Bengtsson, W. Joho, P. Marchand, G. Muelhaupt, L. Rivkin, and A. Streun, Nucl. Instrum. Methods Phys. Res., Sect. A 404, 237 (1998).

[38] J. Bengtsson, SLS Internal Note No. 9/97, http://ados .web.psi.ch/slsnotes/sls0997.pdf.

[39] M. Cornacchia and Y. Chin, Part. Accel. 17, 191 (1985).

[40] L. Emery, in Proceedings of PAC 1989, Chicago IL, USA, 1989, edited by F. Bennett and J. Kopta (IEEE, Piscataway, NJ, 1989), pp. 1225-1227.

[41] L. Emery, in Proceedings of the IEEE Particle Accelerator Conference (APS Beam Physics, San Francisco, California, 1991), pp. 1633-1635.

[42] The original combined-function quadrupole/sextupole was split into two equal focusing quadrupoles that were separated by $150 \mathrm{~mm}$. The $100 \mathrm{~mm}$ long sextupoles were then placed in this gap.

[43] J. Bengtsson, Tracy-2 User's Manual (unpublished).

[44] OPA Lattice Design Code, available at http://people.web .psi.ch/streun/opa/.

[45] L. Nadolski and J. Laskar, Phys. Rev. ST Accel. Beams 6, 114801 (2003).

[46] Diffusion has been defined here as $D=$ $\min \left\{-2 ; \log \sqrt{\left(\Delta \nu_{x}\right)^{2}+\left(\Delta \nu_{y}\right)^{2}}\right\}$, where $\Delta \nu_{x, y}$ denotes the difference between initial tune and tune after 2000 turns.

[47] In fact, in early design studies for the MAX IV storage ring [29], octupoles had already been considered. At that time it was foreseen to include an octupole component in the combined-function quadrupole/sextupole.

[48] In principle, it would be desirable to tune the second-order chromaticity with octupoles rather than with sextupoles (cf. Sec. III). However, one characteristic of an ultralowemittance MBA lattice is that the dispersion becomes very small making the required octupoles unrealistic.

[49] J. Laskar, in Proceedings of the 20th Particle Accelerator Conference, Portland, OR, 2003 (IEEE, New York, 2003), pp. 378-382.

[50] The misalignment and multipole error model is described in detail in [55]. For multipoles we apply what was measured in [56] and for alignment errors we assume $0.1 \mathrm{~mm}$ rms horizontal and vertical misalignment and $0.2 \mathrm{mrad}$ roll error for the girders as well as $0.025 \mathrm{~mm}$ rms horizontal and vertical misalignment and $0.2 \mathrm{mrad}$ roll errors of the individual magnets with respect to the girders. The error distributions are assumed Gaussian with a cutoff at $2 \sigma$.

[51] Actually, the octupoles should be tuned within an overall strategy for restoring the design optics: in a first step gradient strengths are restored with LOCO [57] and the machine is realigned to minimize corrector strength [58]; this can be iterated. As a second step, betatron coupling and vertical dispersion are corrected using skew 
quadrupoles [59]. Finally, in a third step, the nonlinear optics have to be restored by symmetrizing the sextupole pattern [60], possibly exciting auxiliary sextupoles [61], and correcting/optimizing the octupole settings.

[52] M. Sjöström, E. Wallén, M. Eriksson, and L.-J. Lindgren, Nucl. Instrum. Methods Phys. Res., Sect. A 601, 229 (2009).

[53] Y. Cho, A. Rauchas, and A. Wright, in Proceedings of the Seventh National Particle Accelerator Conference, Chicago, 1977, pp. 1563-1564 [IEEE Trans. Nucl. Sci. 24, 3 (1977)].

[54] D. Einfeld et al., in Proceedings of EPAC 1996, Sitges, Spain, 1996 (Ref. [21]).

[55] MAX IV Detailed Design Report, available for download at http://www.maxlab.lu.se/maxlab/max4/index.html.

[56] E. I. Antokhin, I. N. Churkin, V. V. Demenev, O. B. Golubenko, V.N. Korchuganov, A. B. Ogurtsov, E.P.
Semenov, A. G. Steshov, S. F. Mikhailov, L. Rivkin, Ch. Vollenweider, and J. A. Zichy, in Proceedings of the Asian Particle Accelerator Conference, Beijing, 2001 (Institute of High Energy, Physics, Beijing, 2001), p. 209-211.

[57] J. Safranek, Nucl. Instrum. Methods Phys. Res., Sect. A 388, 27 (1997).

[58] M. Sjöström, E. Wallén, M. Eriksson, and L.-J. Lindgren, Nucl. Instrum. Methods Phys. Res., Sect. A 597, 170 (2008).

[59] A. Andersson, M. Böge, A. Lüdeke, and A. Streun, in Proceedings of the 11th European Particle Accelerator Conference, Genoa, 2008 (Ref. [34]), pp. 1983-1985.

[60] R. Bartolini, I. P. S. Martin, J. H. Rowland, P. Kuske, and F. Schmidt, Phys. Rev. ST Accel. Beams 11, 104002 (2008).

[61] M. Böge, A. Lüdeke, and A. Streun, in Proceedings of the 23rd Particle Accelerator Conference, Vancouver, Canada, 2009 (IEEE, Piscataway, NJ, 2009). 